\title{
Deep saturation of a Cerenkov wakefield amplified by an active medium
}

\author{
Z. Toroker and L. Schächter \\ Department of Electrical Engineering, Technion Israel Institute of Technology, Haifa 32000, Israel
}

(Received 25 January 2015; published 7 July 2015)

\begin{abstract}
A trigger bunch of electrons traveling inside or in the vicinity of a dielectric medium generates a Cerenkov wake. If the dielectric medium is active, a small fraction of the spectrum of the wake is amplified and far behind the trigger bunch where the active medium is fully depleted, the amplitude and the phase of the wake are virtually constant. In this range, a second bunch of electrons trailing behind the trigger bunch can be accelerated. For optimal operation, the trigger bunch should be density modulated at the resonant frequency of the medium. However, we demonstrate that even if the bunch is uniform along many wavelengths we may still take advantage of the saturation characteristics to obtain conditions adequate for acceleration. Further we demonstrate that for large enough number of electrons it is possible to have a coherent amplified wake after a saturation length which is determined analytically and tested numerically. In addition, we show that almost $100 \%$ of the stored energy in the active medium can be transferred to the acceleration of the trailing bunch electrons. The relatively large energy spread due to the beam loading is well suited to a medical accelerator. When the beam loading is weak, the gradient is virtually constant but the acceleration efficiency drops to about $2 \%$ for typical parameters.
\end{abstract}

DOI: 10.1103/PhysRevSTAB.18.071301

PACS numbers: 29.20.-c, 29.27.-a, 41.60.-m, 41.75.-i

\section{INTRODUCTION}

Electron acceleration by radio-frequency linear accelerator (rf linac) is the most developed technique that facilitates the generation of a small emittance and energy spread beams of electrons [1-3]. It relies on a single transverse-magnetic (TM) mode which is excited in a metallic circular waveguide and is designed to propagate at the speed of light in vacuum yet it possesses a longitudinal component of the electric field. Since the major limitation of $\mathrm{rf}$ linacs is breakdown, the maximum gradient is of the order of tens of $\mathrm{MV} / \mathrm{m}$ and as a result, a few km's long machine is required for accelerating e-beams to energy of ten's of GeV. The goal of all the new advanced acceleration concepts is to reduce significantly this length by enhancing the gradient while keeping the emittance and the energy-spread as low as possible.

In the past few decades with the advent of high-intensity laser [4] and micro-electronics technologies there is an effort to accelerate electrons to $\mathrm{GeV}$ in a table-top structure. As of today, the record gradients were achieved in the plasma based schemes $[5,6]$ and dielectric based accelerator [7]. In the former, an intense laser pulse is injected into a plasma to generate a wakefield of hundreds $\mathrm{GV} / \mathrm{m}$. The plasma wake, which trails behind the laser pulse can accelerate electrons. In fact, the record energy transferred so far to a bunch of electrons was achieved when the

Published by the American Physical Society under the terms of the Creative Commons Attribution 3.0 License. Further distribution of this work must maintain attribution to the author(s) and the published article's title, journal citation, and DOI. plasma wake was induced by an intense electron bunch [8]. In dielectric based accelerator, it has been demonstrated that a bunch of electrons can excite Cerenkov wake of hundreds $\mathrm{MV} / \mathrm{m}$ [7].

Another technique of electron acceleration relies on transferring energy stored from active medium (AM) to a bunch of electrons $[9,10]$. In the first approach of this technique, spatially modulated electron bunch with periodicity equal to the resonance wavelength of the AM is injected into effectively boundless structure. That bunch generates a wakefield which is amplified by the AM. As a result, the amplified wake directly accelerates the electron bunch. This approach is effective below the Cerenkov condition and it has been demonstrated in an experiment performed at Brookhaven National Laboratory-Accelerator Test Facility [11], in which a density modulated bunch with energy of $45 \mathrm{MeV}$ gained energy of $200 \mathrm{keV}$ from active $\mathrm{CO}_{2}$ gas mixture.

In the second approach considered here electron bunch is accelerated by an amplified Cerenkov wake inside a bounded AM [12]. More specifically, a trigger bunch of electrons travels inside a cylindrical waveguide filled with AM generates TM modes of Cerenkov wake. When the single mode resonance condition is satisfied, only a single TM mode of Cerenkov wake is amplified until full depletion of the population inversion density (PID) occurs. At this point the effective gain is reduced to zero and the wake reaches saturation. Depending on the AM parameters the wake can reach at saturation values as high as $\mathrm{GV} / \mathrm{m}$ a few $\mathrm{cm}$ 's after the trigger bunch. In the region where saturation is reached, a trailing train of bunches may be accelerated by the amplified Cerenkov wake for schematic, see Fig. 1. It is interesting to note that this paradigm resembles the two beam accelerator (TBA) with one major 


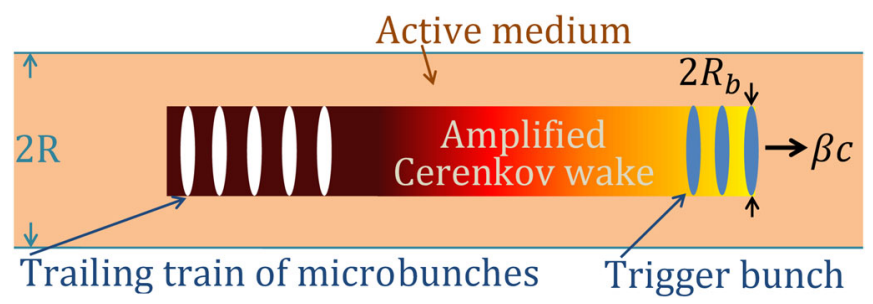

FIG. 1. Schematic description of the AM-TBA for accelerating a trailing train of microbunches that is located at the saturation region.

difference. In the latter, the energy that generates the wake used to accelerate the trailing bunch is stored in the driving bunch. In our case, the energy is stored in the AM.

In previous work we have formulated the nonlinear dynamics of the wake and as an example we considered the case of modulated trigger bunch [13]. Several important analytical expressions have been established: single mode resonance condition, the saturation length, and the saturation value. Our numerical simulations showed that optimal performance can be achieved for large waveguide radius filled with initially high PID and trigger bunch that travels at velocity slightly above the Cerenkov velocity. Electrons' distribution is described by a continuous and deterministic function. In the present study we investigate the wake dynamics for discrete distribution of electrons. We investigate the acceleration of a trailing bunch from the perspective of efficiency, energy spread, amplitude, and phase stability as well as beam loading.

The article is organized as follows: Sec. II presents the dynamics equations that describe the Cerenkov wake amplification in AM TBA with a discrete distribution of the electrons. In Sec. III we summarize the previously analytically calculated results from Ref. [13] as well as additional results from the linear and nonlinear theory brought in detail in Appendices A-D. Specifically, the wake amplitude in the linear regime generated by the trigger train bunch is calculated for passive (Appendix A) and active (Appendix B) media. A detailed calculation of the saturation length is given in Appendix $\mathrm{C}$ and the efficiency of the AM TBA with beam loading is given in Appendix D. In Sec. IV we present simulation results of wake amplification dynamics for a trigger bunch described by discrete distribution of electrons. The phase of the wake and the saturation length are explored numerically for various number of electrons in the microbunch and number of microbunches. In Sec. V we study the energy-conversion efficiency and emittance considering the enhanced wake and the trailing bunch; the results are compared with our analytical formulas from Appendixes D and E.

\section{FORMULATION OF THE PROBLEM}

The dynamics of the wake and the AM inside a cylindrical metallic waveguide of radius $R$ filled with
AM can be described by three sets of equations [13]: Maxwell's equations

$$
\begin{aligned}
\frac{\partial \bar{E}_{z, s}}{\partial \tau}+i \bar{\omega}_{0} \bar{E}_{z, s}= & \frac{\bar{k}_{s}}{2 \sqrt{\epsilon_{r}}} \bar{E}_{+, s}+\frac{\bar{k}_{s}}{2 \sqrt{\epsilon_{r}}} \bar{E}_{-, s} \\
& +\frac{2}{\varepsilon_{r}} \bar{P}_{z, s}-\frac{2}{\varepsilon_{r}} \bar{J}_{s} f \\
\frac{\partial \bar{E}_{+, s}}{\partial \tau}+i \bar{\omega}_{0} \bar{E}_{+, s}= & -\frac{\bar{k}_{s}}{\sqrt{\epsilon_{r}} \Delta \varepsilon_{-}} \bar{E}_{z, s}+\frac{2}{\varepsilon_{r} \Delta \varepsilon_{-}} \bar{P}_{r, s} \\
\frac{\partial \bar{E}_{-, s}}{\partial \tau}+i \bar{\omega}_{0} \bar{E}_{-, s}= & -\frac{\bar{k}_{s}}{\sqrt{\epsilon_{r}} \Delta \varepsilon_{+}} \bar{E}_{z, s}-\frac{2}{\varepsilon_{r} \Delta \varepsilon_{+}} \bar{P}_{r, s} .
\end{aligned}
$$

Polarization dynamics

$$
\begin{gathered}
\frac{\partial \bar{P}_{z, s}}{\partial \tau}+\frac{\Delta \bar{\omega}}{2} \bar{P}_{z, s}=\varepsilon_{r} \bar{n} \bar{E}_{z, s} \\
\frac{\partial \bar{P}_{r, s}}{\partial \tau}+\frac{\Delta \bar{\omega}}{2} \bar{P}_{r, s}=\frac{1}{2} \varepsilon_{r} \bar{n} \bar{E}_{+, s}-\frac{1}{2} \varepsilon_{r} \bar{n} \bar{E}_{-, s}
\end{gathered}
$$

and the equation that describes the PID

$$
\begin{aligned}
\frac{\partial \bar{n}}{\partial \tau}= & -\frac{1}{4} \sum_{s}\left[2 \bar{E}_{z, s}^{*} \bar{P}_{z, s}+2 \bar{E}_{z, s} \bar{P}_{z, s}^{*}\right. \\
& \left.+\left(\bar{E}_{+, s}-\bar{E}_{-, s}\right) \bar{P}_{r, s}^{*}+\left(\bar{E}_{+, s}^{*}-\bar{E}_{-, s}^{*}\right) \bar{P}_{r, s}\right] .
\end{aligned}
$$

Here we assumed that the electrons move with a velocity $\beta c$, we denote $\tau=T \omega_{A}$, where $T=t-z / \beta c, t$, and $z$ are the time and the spatial coordinates. Also, $\omega_{A}=\omega_{p} /\left(2 \sqrt{\epsilon_{r}}\right)$, where $\omega_{p}=\omega_{0} \sqrt{2 n_{0} \mu^{2} / \epsilon_{0} \hbar \omega_{0}}$ is the "plasma frequency" of the AM. An AM with resonance frequency $\omega_{0}$ has initial PID of $n_{0}$, and average dipole moment of $\mu=\mu_{12} / \sqrt{3}$, where $\mu_{12}$ is the dipole moment. Also, the normalized AM resonance bandwidth is $\Delta \bar{\omega}=\Delta \omega / \omega_{A}$. The parameter $\hbar$ is the reduced Planck constant, $\epsilon_{0}$ is the vacuum permittivity, and $\epsilon_{r}$ is the dielectric constant of the medium excluding the population inversion dynamics. Here, it is assumed that $\epsilon_{r}$ is frequency independent. Note that the plasma frequency of the AM is associated with the small signal gain (with respect to the amplitude), $\alpha$, through $\omega_{p}=\sqrt{2 c \Delta \omega \alpha \sqrt{\epsilon_{r}}}$.

The radial wave number of the wake is $k_{s}=p_{s} / R$, where $p_{s}$ is defined through $J_{0}\left(p_{s}\right)=0(s=1,2,3, .$.$) .$ The normalized electric field envelopes, $\bar{E}_{z, s}$ and $\bar{E}_{r, s}$ are normalized with $E_{0}=\left[1 / J_{1}\left(p_{s}\right)\right] \sqrt{\hbar \omega_{0} n_{0} / 2 \epsilon_{0}}$ and the magnetic field envelope $\bar{H}_{s}$ is normalized with $E_{0} /\left(\mu_{0} c\right)$, where $\mu_{0}$ is the vacuum permeability. In addition, $\quad \bar{E}_{ \pm, s}=\bar{H}_{s} / \sqrt{\epsilon_{r}} \pm \bar{E}_{r, s}, \quad \Delta \epsilon_{ \pm}=1 \pm 1 / \beta \sqrt{\epsilon_{r}}$, 
$\bar{\omega}_{0}=\omega_{0} / \omega_{A}$, and $\bar{k}_{s}=k_{s} c / \omega_{A}$. The polarization field envelopes, $\bar{P}_{z, s}$ and $\bar{P}_{r, s}$, are normalized with $i \mu n_{0} / \sqrt{\epsilon_{r}} J_{1}\left(p_{s}\right)$ and the normalized PID, $\bar{n}$, is measured in units of $n_{0}$.

In this model the normalized bunch current is $2 \bar{J}_{s} f / \epsilon_{r}$, where $\bar{J}_{s}=\left(I_{0} / \pi R^{2}\right)\left[J_{c}\left(\chi_{s}\right) / J_{1}\left(p_{s}\right)\right]\left(\sqrt{\epsilon_{r}} / \omega_{0} \mu n_{0}\right), \chi_{s}=$ $k_{s} R_{b}, R_{b}$ is the electron bunch radius, $J_{c}(x) \equiv 2 J_{1}(x) / x$, and $f=f(\tau)$ describes the electron bunch profile in the longitudinal direction. The current bunch can be described by modulated current in which the bunch profile, $f(\tau)$ is a continuous function in the bunch location and $I_{0}=-e N^{(\mathrm{tb})} \beta c / L_{b}$, where $N^{(\mathrm{tb})}$ is the number of the macro-particles that consist of the bunch; $L_{b}$ is the bunch length. More specifically, in Ref. [13] the profile is $f\left(\tau_{0}<\right.$ $\left.\tau<\tau_{1}\right)=1$ and zero otherwise where $\tau_{0}$ is the injection time of the bunch, $\tau_{1}=\tau_{0}+\bar{L}_{b}$, and $\bar{L}_{b}=L_{b} \omega_{A} / \beta c$ is the normalized bunch length. In this paper, we explore mainly the case that the current is described by discrete microbunches. In this case the trigger bunch consists of $M^{(\mathrm{tg})}$ microbunches with $N^{(\mathrm{tg})}$ electrons in each of them. The microbunches are separated a distance of one resonance wavelength. Hence, the profile for discrete electron bunch is $\quad f(\tau)=\sum_{\nu=1}^{M^{(\mathrm{gg})}} \sum_{m=1}^{N^{(\mathrm{g})}} \delta\left[\tau-\tau_{m}-2 \pi / \bar{\omega}_{0}(\nu-1)\right] e^{-i \bar{\omega}_{0} \tau}$ and $I_{0}=-e \omega_{A}$.

The set of equations given in Eqs. (1)-(6) conserves energy,

$$
\frac{\partial \bar{W}_{\mathrm{tot}}}{\partial \tau}=0
$$

where the normalized total energy is

$$
\bar{W}_{\mathrm{tot}}=\bar{W}_{n}+\sum_{s=1}^{\infty}\left[\bar{W}_{s}^{(\mathrm{EM}, \mathrm{lo})}+\bar{W}_{s}^{(\mathrm{EM}, \mathrm{tr})}+\bar{W}_{s}^{(B)}\right],
$$

$\bar{W}_{n}=\bar{n}$ is the energy stored in the $\mathrm{AM}, \bar{W}_{s}^{(\mathrm{EM}, \mathrm{lo})}=$ $\epsilon_{r}\left(\left|\bar{E}_{z, s}\right|^{2}+\bar{E}_{z, s}^{2} e^{i 2 \bar{\omega}_{0} \tau} / 2+\bar{E}_{z, s}^{* 2} e^{-i 2 \bar{\omega}_{0} \tau} / 2\right) / 4$ is the energy density associated with the longitudinal electric field, and $\bar{W}_{s}^{(\mathrm{EM}, \text { tr })}=\epsilon_{r}\left[\Delta \epsilon_{-}\left(\left|\bar{E}_{+, s}\right|^{2}+\bar{E}_{+, s}^{2} e^{i 2 \bar{\omega}_{0} \tau} / 2+\bar{E}_{+, s}^{* 2} e^{-i 2 \bar{\omega}_{0} \tau} / 2\right)\right.$ $\left.+\Delta \epsilon_{+}\left(\left|\bar{E}_{-, s}\right|^{2}+\bar{E}_{-, s}^{2} e^{i 2 \bar{\omega}_{0} \tau} / 2+\bar{E}_{-, s}^{* 2} e^{-i 2 \bar{\omega}_{0} \tau} / 2\right)\right] / 8$ is the transverse components counterpart.

Note that $\epsilon_{c}=\Delta \epsilon_{+} \Delta \epsilon_{-}=1-1 /\left(\beta^{2} \epsilon_{r}\right)$ and is associated with the Cerenkov parameter in Ref. [12] by $\epsilon_{c}=\bar{\epsilon} / \epsilon_{r}$. In addition, $\bar{W}_{s}^{(B)}=\bar{J}_{s} \int_{0}^{\tau} d \tau^{\prime}\left[f^{*}\left(\tau^{\prime}\right) \bar{E}_{z, s}\left(\tau^{\prime}\right)+f\left(\tau^{\prime}\right) \bar{E}_{z, s}^{*}\left(\tau^{\prime}\right)\right.$ $\left.+f\left(\tau^{\prime}\right) \bar{E}_{z, s}\left(\tau^{\prime}\right)+f^{*}\left(\tau^{\prime}\right) \bar{E}_{z, s}^{*}\left(\tau^{\prime}\right)\right] / 2$ is the energy of the bunch.

In the framework of this model it was assumed that: (i) the system is azimuthally symmetric, (ii) collisions of the electrons with the medium may be ignored, (iii) $\mathrm{Ohm}$ loss of the electromagnetic field can be discarded, and (iv) the PID is uniform in the radial direction.

\section{ANALYTIC ASSESSMENTS}

In the framework of this section we recapitulate the most important analytical results presented in detail in [13] as well as recently developed results; this summary is limited to results that are of importance to the present study. In case of zero gain, a wave which oscillates at the resonance frequency of the medium and it has a phase velocity equal to the velocity of the electron, satisfies the following dispersion relation

$$
\left(\frac{\omega_{0} \sqrt{\epsilon_{r}}}{c}\right)^{2}-\left(\frac{p_{s_{0}}}{R}\right)^{2}=\left(\frac{\omega_{0}}{\beta c}\right)^{2},
$$

where $s=s_{0}$ is the resonance mode number. The time duration needed to the amplified wake to reach saturation is

$$
t_{\mathrm{sat}}=t_{d}+t_{r}=\frac{1}{\delta \omega} \ln \left[\frac{2\left|E_{\mathrm{sat}}\right|}{\left|E_{z, s_{0}}\left(T_{1}\right)\right|}\right]+\frac{20}{\Delta \omega},
$$

where

$$
\delta \omega=\frac{\Delta \omega}{4}\left[-1+\sqrt{1+\frac{8 c \alpha \sqrt{\epsilon_{r}}}{\bar{\epsilon} \Delta \omega}}\right]
$$

is the linear growth rate of the wake and $E_{z, s_{0}}\left(T_{1}\right)$ is the wake immediately after the trigger bunch. The parameter $t_{d}$ is the first time the PID reaches zero namely full depletion and the parameter $t_{r}$ is the relaxation time of the PID in the nonlinear regime of amplification due to the Rabi oscillation (for more details see Ref. [13]). The absolute value of the saturated wake, $\left|E_{\mathrm{sat}}\right|=\left|E_{z, s_{0}}\left(t \geq t_{\mathrm{sat}}\right)\right|$, is found from energy conservation

$$
\epsilon_{0} \epsilon_{r} \frac{\left|J_{1}\left(p_{s_{0}}\right) E_{\mathrm{sat}}\right|^{2}}{2}=\frac{\hbar \omega_{0} n_{0}}{2},
$$

where it is assumed that $E_{ \pm, s_{0}}=i\left(k_{s_{0}} / \sqrt{\epsilon_{r}} \omega_{0} \Delta \epsilon_{\mp}\right) E_{z, s_{0}}$. One comment is in place here: the smaller the Cerenkov slippage $\left(\bar{\epsilon}=\epsilon_{r}-\beta^{-2}\right)$ the larger the growth rate.

The results shown above are independent on whether the electrons are represented by continuous or discrete distributions. Now, consider a train of $M^{(\mathrm{tg})}$ microbunches with one (resonance) wavelength spacing and the train propagates either in passive or AM. Each microbunch consists of $N^{(\mathrm{tg})}$ electrons that are randomly distributed over a length $\Delta z<\lambda_{0}$ corresponding to initial phase distribution (on the resonant mode $-s_{0}$ ) $2 \psi=\omega_{0} \Delta z / \beta c$; the super script $(\operatorname{tg})$ indicates that the parameter describes the trigger bunch.

For a large number of experiments, the averaged wakefield amplitude and its standard deviation immediately after the last microbunch were calculated in Appendices A and B. The results are summarized in Table I. 
TABLE I. Averaged and standard deviation of the wake complex amplitude in passive and active media. Here, the initial phase-spread of the electrons at the resonance mode $s_{0}$ is $2 \psi=\omega_{0} \Delta z / \beta c$, where $\Delta z$ corresponding to initial length of electrons locations. For the results regarding the passive medium it was assumed that the electrons are uniformly distributed. For the AM, the results are the same for both uniform and normal distributions but with different $F$ function.

\begin{tabular}{lcc}
\hline \hline & Passive (Appendix A) & Active (Appendix B) \\
\hline$\frac{\left\langle E_{z, s_{0}}(r=0, T)\right\rangle}{E_{s_{0}}}$ & $\operatorname{sinc}(\psi)$ & $e^{\delta \omega T} G F$ \\
$\frac{\Delta E_{z, s_{0}}(r=0, T)}{\left|E_{s_{0}}\right|}$ & $\frac{\sqrt{1-\operatorname{sinc}^{2}(\psi)}}{\sqrt{N^{(\mathrm{tg})}}}$ & $\frac{\sqrt{1-|F|^{2}}}{\sqrt{N^{(\mathrm{gg})}}} e^{\delta \omega T} G$ \\
$\frac{\Delta E_{z, s_{0}}(r=0, T)}{\left|\left\langle E_{z, s_{0}}(r=0, T)\right\rangle\right|}$ & $\frac{\sqrt{1-\operatorname{sinc}^{2}(\psi)}}{\sqrt{N^{(\mathrm{tg})}|\operatorname{sinc}(\psi)|}}$ & $\frac{\sqrt{1-|F|^{2}}}{\sqrt{N^{(\mathrm{tg})}|F|}}$ \\
\hline \hline
\end{tabular}

The averaged complex amplitude and its standard deviation are normalized with $E_{S_{0}}=\left(e N^{(\mathrm{tg})} M^{(\mathrm{tg})} /\right.$ $\left.\epsilon_{0} \epsilon_{r} \pi R^{2}\right)\left[J_{c}\left(\chi_{s_{0}}\right) / J_{1}^{2}\left(p_{s_{0}}\right)\right]$, which is the wakefield amplitude for a pointlike electron bunch $\left(\Delta_{z}=0\right)$ in a passive medium. In addition, the function $G$ is given by

$$
\begin{aligned}
G\left(q, u, M^{(\mathrm{tg})}\right) \equiv & \frac{1}{2}\left(1+\frac{1}{1+4 q}\right) \\
& \times e^{\pi u\left(M^{(\mathrm{g})}-1\right)} \frac{\sinh \left(\pi u M^{(\mathrm{tg})}\right)}{M^{(\mathrm{tg})} \sinh (\pi u)} .
\end{aligned}
$$

For a microbunch consisting of electrons that are distributed uniformly the function $F$ reads

$$
F(u, \psi) \equiv \operatorname{sinc}(\psi-i u),
$$

where $q=\delta \omega / \Delta \omega$ and $u=\delta \omega / \omega_{0}$ are the normalized growth rate with respect to the resonance frequency and the AM bandwidth, respectively. However, for a normal distribution with the same variance of the uniform distribution the function $F$ is replaced with $F^{(\mathrm{N})}=\exp \left[-\sigma^{2}\left(1-u^{2}\right) / 2+i \sigma u\right]$, where $\sigma=2 \psi / \sqrt{12}$.

First note, as expected, that in the limit of zero gain $(\delta \omega=0)$ the results of the AM converge to those of the passive medium. For $\delta \omega>0$ the amplitude of the wake in the $\mathrm{AM}$ is growing exponential as $\exp (\delta \omega T)$ in the linear regime, whereas in the passive medium it remains constant (confined Cerenkov radiation). As can be seen from Table I the difference between the averaged wake in passive and active media is not only in the absolute value but also in the phase since $F(u, \psi)$ is a complex function. In a passive medium the phase does not depend on the initial spread $\psi$. However, in an AM the phase depends on both the growth rate and the initial spread of the electrons. Another important difference is that for a uniform beam (initial spread of $2 \psi=2 \pi$ ) the averaged wake amplitude in a passive medium is zero, while in an AM is larger than zero.
Specifically, the averaged wake complex amplitude in an $\mathrm{AM}$ is

$$
\frac{\left\langle E_{z, s_{0}}^{\text {(act) }}(r=0, T)\right\rangle}{E_{S_{0}}}=e^{\delta \omega T} G \frac{i \sinh (\pi u)}{\pi(1-i u)}
$$

thus keeping in mind that within a good approximation $1-i u \approx 1$, the phase of the wake is shifted by $\pi / 2$. Subsequently, we discuss the phase shift of the wake for initially uniformly and normally distributions of electrons in more detail in Sec. IV.

For e-beam acceleration it is important not only that the averaged wake amplitude will be large but also to have small standard deviation in order to minimize the jitter experienced by the trailing bunch. Thus, we require that the signal-to-noise ratio, $\left|\left\langle E_{z, s_{0}}(r=0, T)\right\rangle\right| / \Delta E_{z, s_{0}}(r=0, T)$, to be as large as possible. In passive medium (with a uniform initial distribution of the electrons) this requirement is equivalent to

$$
N^{(\mathrm{tg})} \geq N_{\mathrm{thr}}^{(\mathrm{pas})} \equiv \frac{1-\operatorname{sinc}^{2}(\psi)}{\operatorname{sinc}^{2}(\psi)}
$$

and in $\mathrm{AM}$

$$
N^{(\mathrm{tg})} \geq N_{\mathrm{thr}}^{(\mathrm{act})} \equiv \frac{1-|F(u, \psi)|^{2}}{|F(u, \psi)|^{2}} .
$$

With the electric field immediately after the last trigger microbunch determined, we can proceed to an assessment of the saturation relying primarily on energy conservation. The detailed calculation is presented in Appendix C. For a sufficiently large number of experiments the average saturation time is

$$
t_{\mathrm{sat}}=\frac{1}{\delta \omega} \ln \left(\sqrt{\frac{U_{a}}{U_{0}}}\right)+\frac{20}{\Delta \omega},
$$

where the initial energy stored per unit length in the AM, $U_{a}$, and the electromagnetic energy per unit length generated by the trigger bunch immediately after the end of the bunch, $U_{0}$, are given in Appendix B.

After establishing the optimal conditions on the parameters of the trigger bunch in order to generate a wake amplitude with minimum standard deviation, we proceed to calculate the efficiency of our system in the presence of a trailing train of microbunches. The natural place to accelerate the latter is in the saturation region, where the wakefield reaches its highest value and in the absence of the accelerated (trailing) bunch, its amplitude is uniform. The efficiency of our structure is defined by

$$
\eta=\frac{W_{b} L}{W_{\mathrm{em}}\left(t=t_{\mathrm{sat}}\right)\left(L+L_{\mathrm{sat}}\right)} \simeq \frac{W_{b}}{\frac{1}{2} \hbar \omega_{0} \pi R^{2} n_{0}},
$$


where $W_{b}$ is the energy exchange (per unit length), $L_{\text {sat }}=$ $\beta c t_{\text {sat }}$ is the saturation length, and $L$ is the propagating distance of the accelerating bunch which is assumed to be larger than the saturation length. In the case of beam loading in the saturation regime the energy exchange (or energy gain) is of the form of

$$
W_{b}=W_{b, 1} N^{(\mathrm{tl})}\left(1-N^{(\mathrm{tl})} f_{\mathrm{col}}\right),
$$

where $W_{b, 1}$ is the energy transfer in case of a single electron per microbunch, $f_{\text {col }}$ is the collective-effect coefficient; both quantities are defined in Appendix D; a similar expression was developed for conventional rf-linacs [14]. For a small number of electrons $\left[N^{(\mathrm{tl})}\right]$ the wake amplitude is almost unchanged but the global energy exchange is weak. If however, the number of accelerated electrons is elevated, $W_{b, 1}\left(1-N^{(\mathrm{tl})} f_{\text {col }}\right)$ the effective energy-exchange may approach zero because of the beam-loading effect thus again, weak energy exchange. In between, there is an optimal number of electrons that may be readily shown to be maximum efficiency $N^{(\mathrm{tl})} \simeq 1 / 2 f_{\text {col }}$.

As demonstrated in Appendix D, almost 100\% efficiency can be achieved for initial small spread of each microbunch, $\left(\Delta z^{(\mathrm{tl})} \ll \lambda_{0}\right)$, and for large number of microbunches $M^{(\mathrm{tl})} \gg 1$ however, the high efficiency is accompanied by large energy spread since the field gradient is not constant as it will be shown in Sec. VA. When low efficiencies can be tolerated, we present in Secs. VB and $\mathrm{VC}$ two additional configurations to obtain a high energy gain with a low energy spread.

\section{WAKE DYNAMICS}

In this section we examine the nonlinear dynamics of the wake that is generated by a bunch of discrete electrons in an active $\mathrm{CO}_{2}$ gas mixture with the same structure parameters as in the linear analysis presented in Refs. $[12,15]$ but with a bunch radius of $R_{b}=30 \mu \mathrm{m}$. We begin with studying the dynamics of the wake triggered by a single electron. Subsequently, the number of electrons is increased and they are randomly distributed with either uniform or normal distributions over a length of one and a half resonance wavelengths $(2 \psi=3 \pi)$. As will be shown, the phase-spread of the wake can be significantly reduced for large enough number of electrons; a feature which is crucial for accelerating a second trailing bunch. A similar impact has the number of electrons in the bunch on the saturation length.

\section{A. Number of electrons in trigger bunch}

First let us consider the case of a single electron trigger bunch. In this case Fig. 2 shows the dynamics of the wake (black solid curve) and the PID. It reveals that even a single electron can generate a wake that will reach saturation in a $\mathrm{cm}$ scale length. As a comparison, Fig. 2 shows also the wake amplification by a modulated trigger bunch (blue

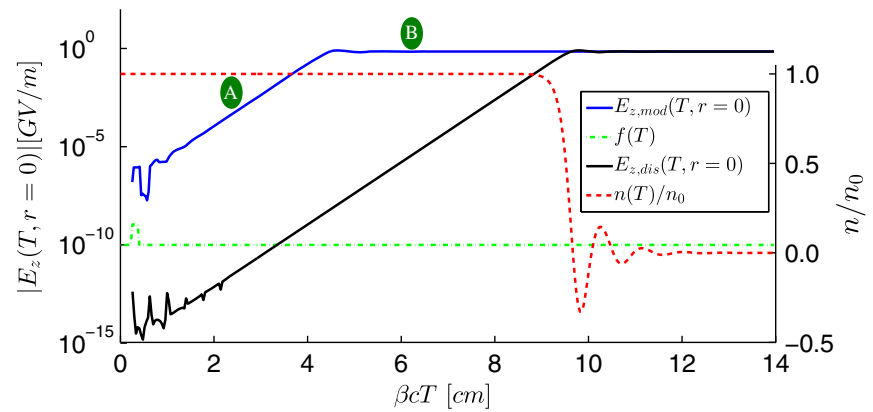

FIG. 2. Wake comparison between the modulated trigger bunch (blue solid curve) with the single electron trigger bunch (black solid curve). The green dotted curve shows the location of the trigger bunch and the dashed curve shows the PID dynamics. The ellipses A and B show the locations of the accelerated second trailing train bunch that will be considered in the acceleration regimes section $(\mathrm{V})$.

solid curve) unveiling that the wake reaches saturation within $5 \mathrm{~cm}$ behind the bunch, whereas for a single electron the saturation occurs within $10 \mathrm{~cm}$ behind the trigger electron. In addition, for wake dynamics as the former the ellipses A and B show the locations of the accelerated second trailing train bunch that will be considered in the acceleration regimes section $(\mathrm{V})$.

Though a single electron can generate strong wake within a length of a few cm's, in practice the trigger bunch is composed of large number of electrons $\left(N^{(\mathrm{tg})} \gg 1\right)$. Here, we assume that each injected trigger bunch is composed of $N^{(\mathrm{tg})}$ electrons that are randomly distributed with either uniform or normal distributions over a length of one and a half resonance wavelengths $(2 \psi=3 \pi$ and $M^{(\mathrm{tg})}=1$ ), namely a length of $10.6 \times 1.5=15.9 \mu \mathrm{m}$ in our case of $\mathrm{CO}_{2}$ gas mixture.
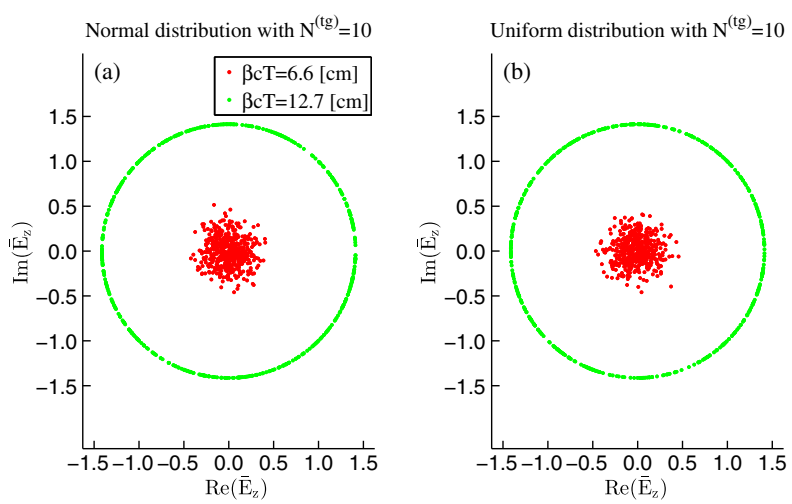

FIG. 3. The real part and the imaginary part of the wakefield in the linear regime (at $\beta c T=6.6 \mathrm{~cm}$ ) and in the nonlinear regime (at $\beta c T=12.7 \mathrm{~cm}$ ). The wake is triggered by a bunch consisting of $N^{(\mathrm{tg})}=10$ electrons that are spread either (a) normally or (b) uniformly with standard deviation of one and a half resonance wavelengths $(2 \psi=3 \pi)$. Also, the number of the circles at $\beta c T=$ $6.6 \mathrm{~cm}$ and $\beta c T=12.7 \mathrm{~cm}$ equals to the number of the numerical simulations, $N_{\exp }=500$. 
At saturation, as shown in Fig. 3, the wake amplitude is well determined and can also be calculated from Eq. (12). However, the phase of the wake is not well determined due to the initial random distribution of the electrons. For example, Fig. 3 shows the real and imaginary parts of the wake at the locations $\beta c T=6.6 \mathrm{~cm}$ (linear regime) and $\beta c T=12.7 \mathrm{~cm}$ (saturation regime). Each dot represents the complex wake amplitude for 10 electrons confined in a one and a half (resonance) wavelengths with normal distribution [Fig. 3 (a)] and uniform distribution [Fig. 3 (b)] of initial electrons position. The averaged initial positions of the electrons is $3.5 \lambda_{0}$ corresponding to a phase of $\pi$. Also, for the normal distribution the standard deviation is $\sigma=2 \psi / \sqrt{12} \simeq 0.58 \pi$. Since $N_{\text {exp }}=500$ experiments were performed this is the number of red and green circles. In the linear regime (red) the wake real and imaginary parts values fill a circlelike shape whereas at saturation (green) the wake amplitude is constant but the spread of the wake phase is $2 \pi$. This phase randomness makes this regime impractical for accelerating a second trailing bunch.

However, when the number of electrons in the trigger bunch is increased, for example, to 100 and the electrons are uniformly distributed as is illustrated in Fig. 4(b), the spread of the phase is reduced significantly from $2 \pi$ to $0.11 \pi$, whereas for electrons that are normally distributed the phase-spread of the wake is still $2 \pi$ [Fig. 4(a)]. Hence, in this regime the phase-spread is sensitive to the initial distribution function. This difference will be discussed in more detail in the last part of this subsection.

In the linear regime, as the number of electrons is increased, the wake amplitude is also increased-as indicated by the red circles of Fig. 4(a). For a large number of electrons the wake can reach the nonlinear regime, where the PID and the wake experience Rabi oscillations. Locally, the amplitude may exceed the stable saturation level—as illustrated in Fig. 4(b).

For 10,000 electrons that are either normally or uniformly distributed, the phase-spread is significantly smaller than $2 \pi$ as shown in Fig. 5. Specifically, for the normal distribution
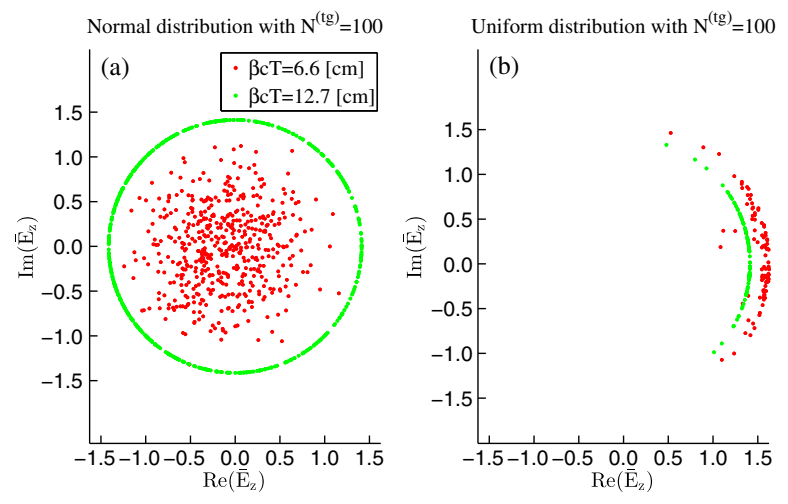

FIG. 4. The same as Fig. 3 but with $N^{(\mathrm{tg})}=100$.
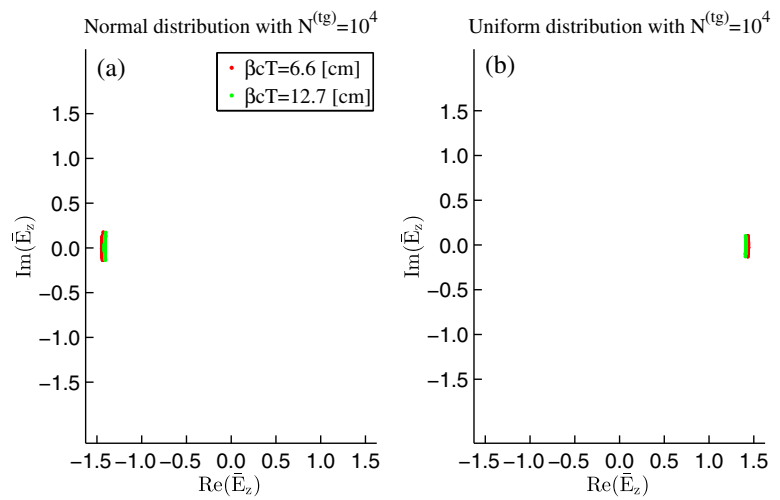

FIG. 5. The same as Fig. 3 but with $N^{(\mathrm{tg})}=10^{4}$.

the phase-spread is $0.0118 \pi$ with an average phase of $\pi$ and for the uniform distribution, the phase-spread is $0.0112 \pi$ with a zero average phase. The reason for the different average phase stems from the different distribution functions. For uniform distribution $F(u, \psi) \simeq-0.22$ and for normal distribution $F(u, \psi) \simeq 0.025$, where $\psi=1.5 \pi$ and $u=6.14 \times 10^{-4}$. In addition, the trigger bunch averaged initial position is $3.5 \lambda_{0}$ corresponding to a phase of $\pi$. Thus, the total phase shift of the wake is 0 rad for the uniform distribution and $\pi$ rad for the normal distribution-results that are in agreement with Fig. 5.

The most important result from Figs. 3-5 is that in both random distributions, by increasing the number of electrons in the trigger bunch, we may reduce significantly the phasespread of the wake. This is crucial for acceleration of a trailing train of bunches. As indicated, this feature is revealed only when the number of the electrons is above a critical value depicted in Fig. 6 for both distributions. In fact, this critical value is the threshold of electron number that was defined in Eq. (17). For various initial electron

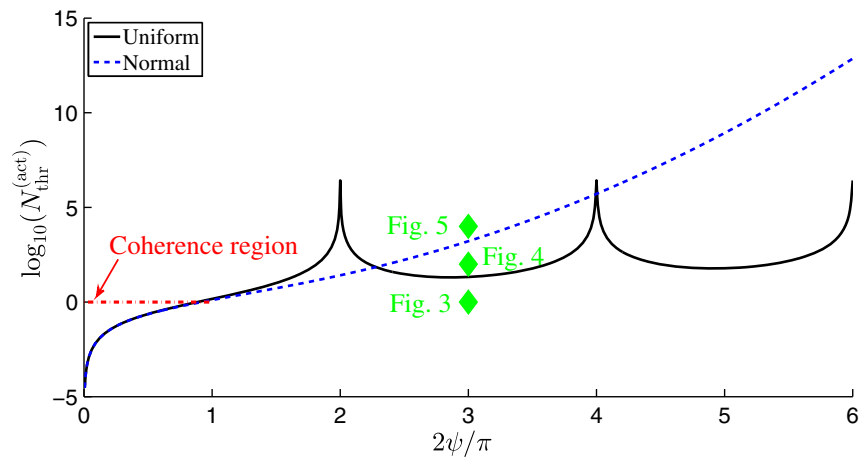

FIG. 6. The threshold of electron number in a microbunch (shown in a logarithmic scale) as function of the phase-spread of the electrons for uniform phase distribution (solid black curve) and for normal phase distribution (blue dashed curve). For the uniform distribution the phase is spread over the range of $(-\psi, \psi)$ with standard deviation of $2 \psi / \sqrt{12}$. For the normal distribution the phase is spread over the range of $(-\infty, \infty)$ with the same standard deviation as the uniform distribution. 
spreads the electron threshold number is depicted in Fig. 6. Clearly, for initial electron phase-spread smaller than about $\pi$ or half resonance wavelength, the electron threshold number is smaller than one, which means that the spread of the wake phase is practically zero for any amount of number of electrons and independent on the distribution function. Hence, in this region the amplified radiation is coherent.

In order to maintain the coherence for larger initial spread of electrons, as their wake is amplified by the AM, the number of electrons should be increased. For example, we previously studied the case of $2 \psi=3 \pi$ for various $N^{(\mathrm{tg})}$ (see Figs. 3-5). In this example the electrons' threshold number for the uniform distribution is 20 and 1590 for the normal distribution. Thus, for $N^{(\mathrm{tg})}=10$ the spread of the wake is expected to be $2 \pi$. For $N^{(\mathrm{tg})}=100$ only the spread of the wake phase is expected to decrease for the uniform distribution and for 10,000 electrons the phase-spread is decreased for both distributions. This explains our choice of parameters in Figs. 3-5.

Our analytical calculations [Eqs. (16) and (17)] may be counterintuitive when compared to self-amplified spontaneous emission (SASE) in lasers where the medium amplifies a homogeneous wave, namely, the photons' phase are also randomly distributed over one resonance wavelength. In other words, the phase-spread is always $2 \pi$.

The major reason for the discrepancy is related to the different character of the homogenous solution of the wave equation and its nonhomogeneous counterpart. While photons set a constraint on the frequency of the radiation $(\omega)$, electrons set a constraint on the phase velocity, $\beta c=\omega / k_{z}$, where $k_{z}$ is the wave number in the longitudinal direction. Thus, at the resonant frequency $\left(\omega=\omega_{0}\right)$ only the longitudinal wave number is complex for photons, whereas for electrons, both the frequency and wave number are complex. Explicitly, photons amplification at resonance is described by $E^{(\mathrm{ph})} \propto$ $\left\langle\exp (\alpha z) \cos \left[\omega_{0}\left(t-z \sqrt{\epsilon_{r}} / c\right)-\phi_{i}\right]\right\rangle_{i}$ wherein $\phi_{i}$ is the phase of the photon when born and for electrons $E^{(\mathrm{el})} \propto\left\langle\exp \left\{\delta \omega\left[t-\left(z-z_{i}\right) / v\right]\right\} \cos \left\{\omega_{0}\left[t-\left(z-z_{i}\right) / v\right]\right\}\right\rangle_{i}$, where $z_{i}(i=1,2,3, \ldots)$ is the initial position of the electron. As a result, at the limit of large number of photons the average field is zero $\left(\left\langle E^{\mathrm{ph}}\right\rangle=0\right)$ whereas in case of nonhomogeneous solutions of the wave equation the average field does not vanish $\left(\left|\left\langle E^{\mathrm{el}}\right\rangle\right|>0\right)$ even if the number of electrons is very large.

The result revealed in Fig. 5, is one of the important results of the present study since it indicates that the AM affects the "relative weight" of electrons at the front comparing to these at the end of the bunch. This asymmetry leads to a nonzero electron field contrary to the photons case which their contribution averages to zero. The implications on the feasibility of utilizing this regime for acceleration is crucial since it indicates that we may start with a uniform distribution yet, the wake immediately after the bunch is not zero and not less important, the jitter of the phase can be minimized by increasing the number of electrons in the bunch.

\section{B. Phase-Spread}

A key parameter of the AM-TBA is the phase-spread of the wakefield. It needs to be as small as possible to enable the acceleration of the trailing bunch in a constant and wellestablished position relative to the trigger bunch. Moreover, a small phase-spread enables staging of our system, namely, it makes possible to accelerate in several stages of active media. We begin with exploring the phase-spread of the wakefield, $\Delta \Psi$, for various number of electrons in the trigger bunch, $N^{(\mathrm{tg})}$, and various initial phase-spread of the electrons, $\psi$, in the trigger bunch.

Figure 7 shows $\Delta \Psi$ as function of the initial electron phase-spread, $2 \psi$, for $N^{(\mathrm{tg})}=10$ (blue diamonds), $N^{(\mathrm{tg})}=$ 100 (red diamonds), and $N^{(\mathrm{tg})}=1000$ (green diamonds); as in the previous case $N_{\text {exp }}=500$ experiments were performed and the phase of the electrons is distributed normally. For initially small spread of the electron phase (less than $\pi$ ) the phase-spread of the wake is smaller than $0.1 \pi$; hence, the wake is coherent and thus adequate for electron acceleration. When, the initial spread of the electrons is increased, larger number of electrons is required to maintain minimal spread of the wake phase. For example, in the case of $2 \psi / \pi=1.5$ the phase-spread is decreased from $0.2 \pi \mathrm{rad}$ to about $0.01 \pi \mathrm{rad}$ when the number of electrons in the microbunch is increased from $N^{(\mathrm{tg})}=10$ to $N^{(\mathrm{tg})}=1,000$. Moreover, our numerical simulation results are also consistent with our analytical

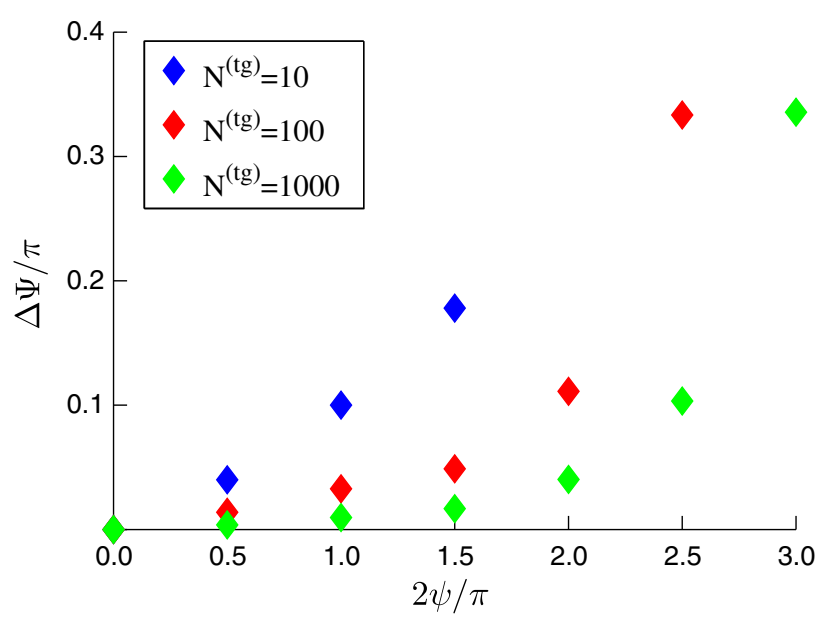

FIG. 7. The dependence of the phase-spread of the wake $(\Delta \Psi)$ on the initial phase distribution of the electrons, $2 \psi$, for $N^{(\mathrm{tg})}=$ 10 (blue diamonds), $N^{(\mathrm{tg})}=100$ (red diamonds), and $N^{(\mathrm{tg})}=$ 1000 (green diamonds). Here the phase-spread of the wake is defined as the standard deviation of the wake phase at $\beta c T=$ $12.7 \mathrm{~cm}$ for 500 numerical simulations ("experiments"). 
calculations of the electron threshold number that is shown in Fig. 6.

\section{Saturation length}

So far we have shown that a sufficiently large number of electrons in the trigger microbunches can suppress the phase-spread of the wake. In this subsection we examine the dependence of the saturation length on the trigger bunch parameters-see Eq. (18).

Figure 8 shows the saturation length as a function of the initial electrons' phase-spread $\psi$ for various trigger bunch parameters and phase that is either distributed uniformly or normally. As an example, for a trigger bunch with $N^{(\mathrm{tg})}=$ $2.8 \times 10^{6}$ and $M^{(\mathrm{tg})}=10$ the saturation length is of the order of $5 \mathrm{~cm}$ for a short spread of electron phase in both uniform (green solid curve) and normal (red dashed curve) distributions of the electrons' phase. However, when the electrons are spread over a length that is above a half the resonance wavelength $(2 \psi>\pi)$, the saturation length is larger than for the pointlike microbunch and strongly dependent on the distribution function (whether uniform or normal). Comparing to the pointlike distribution, the saturation length is increased since for a larger phasespread the wake amplitude is weaker due to a destructive interference.

Increasing the number of electrons for a short spread trigger bunch with $N^{(\mathrm{tg})}=2.8 \times 10^{6}$ and $M^{(\mathrm{tg})}=10$ to $N^{(\mathrm{tg})}=2.8 \times 10^{7}$ with $M^{(\mathrm{tg})}=100$ (black dashed-dotted and blue dotted curves) reduces the saturation length from nearly $5 \mathrm{~cm}$ to $4 \mathrm{~cm}$. Similarly, as in the former trigger bunch, for a larger phase-spread, the saturation length is strongly dependent on the distribution function-whether uniform (black dashed-dotted curve) or normal (blue dotted curve).

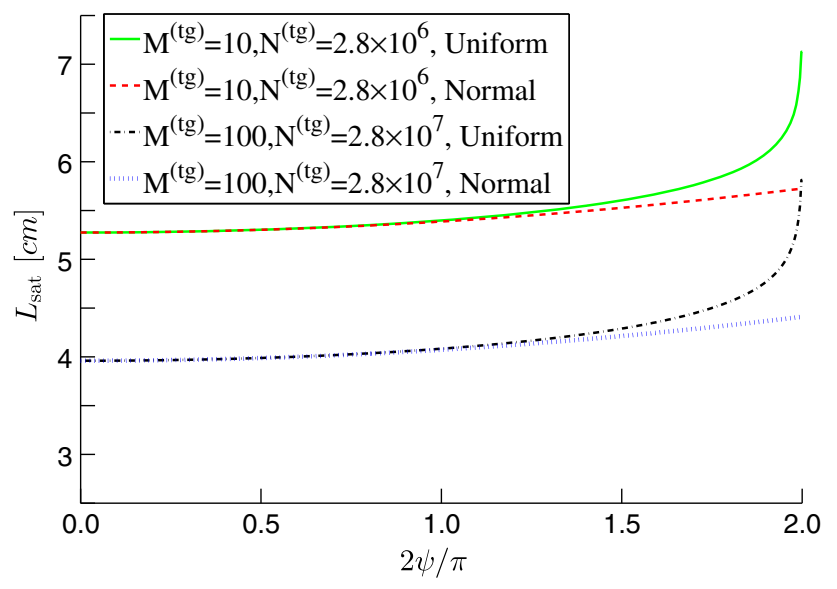

FIG. 8. The dependence of the saturation length [Eq. (18)] on the number of electrons in each microbunch $N^{(\mathrm{tg})}$, the number of microbunches $M^{(\mathrm{tg})}$, and the initial phase-spread of each microbunch $2 \psi / \pi$.
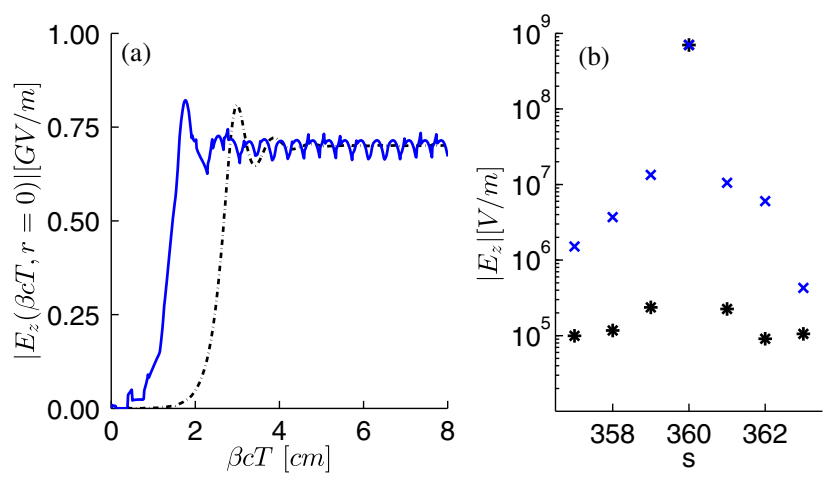

FIG. 9. (a) The wake dynamics for trigger bunch with $M^{(\mathrm{tg})}=$ $100, N^{(\mathrm{tg})}=2.8 \times 10^{7}$ (blue solid curve) and $M^{(\mathrm{tg})}=10, N^{(\mathrm{tg})}=$ $2.8 \times 10^{6}$ (black dashed-dot curve) in the length interval of $0 \leq \beta c T \leq 8 \mathrm{~cm}$. In addition, each microbunch is a pointlike, namely, $\psi=0$. (b) Comparison of the wake spectrum for $M^{(\mathrm{tg})}=$ $100, N^{(\mathrm{tg})}=2.8 \times 10^{7}$ (blue solid curve and blue crosses) with $M^{(\mathrm{tg})}=10, N^{(\mathrm{tg})}=2.8 \times 10^{6}$ (black dashed-dot curve and black asterisks) at $\beta c T=8 \mathrm{~cm}$.

\section{Oscillations}

While increasing the number of trigger electron, $N^{(\mathrm{tg})}$, reduces the saturation length, it also increases dramatically oscillations - as illustrated by Fig. 9(a) for pointlike microbunches $(\psi=0)$. For instance, increasing the number of electrons in a microbunch to $N^{(\mathrm{tg})}=2.8 \times 10^{7}$ with number of microbunches of $M^{(\mathrm{tg})}=100$ results in a nonconstant wake amplitude at saturation. More specifically, Fig. 9(a) compares the wake dynamics from $N^{(\mathrm{tg})}=2.8 \times$ $10^{6}$ and $M^{(\mathrm{tg})}=10$ (black dashed curve) with $N^{(\mathrm{tg})}=$ $2.8 \times 10^{7}$ and $M^{(\mathrm{tg})}=100$ (blue solid curve). As shown, the wake amplitude at saturation oscillates more in the latter case than the former. We also checked that if we increase only the number of microbunches to 100 but keep the number of electrons per microbunch to be $2.8 \times 10^{6}$, the wake amplitude will be nearly constant.

We can comprehend this result keeping in mind that high charge per microbunch and the finite bandwidth of the gain contribute to the intensity of the off resonance modes. In other words, oscillations of the wake at saturation can occur due to interference of the off-resonance modes [Fig. 9(b)]. Consequently, it is important not to increase too much the number of electrons in a microbunch. This is another important result of our study that needs to be taken into account in any practical design.

\section{ACCELERATION REGIMES}

In this section we explore the system's efficiency and its ability to accelerate electrons. After demonstrating that we can minimize the phase-spread of the wake we now proceed to examine in more detail the acceleration process. There are two interrelated perspectives that need to be considered: energy conversion efficiency and energy-spread due to 
beam loading. The natural place to locate a train of trailing microbunches in order to accelerate the electrons is in the region where the wake has reached saturation. In Appendix D, we calculate the efficiency and it is demonstrated that, as a function of the electrons in the microbunch, it may reach a maximum, $\eta^{(\max )}$, [Eq. (D16)]; for a pointlike microbunch $\left(\Delta_{z} \ll \lambda_{0}\right)$ and for large number of microbunches $M^{(\mathrm{tb})} \gg 1$ the analytic expression is relatively simple. In the following subsections both the trigger and the trailing bunches consist of a pointlike microbunches.

\section{A. High efficiency with large energy spread}

As a first example, we investigate the high-efficiency regime. Close to $100 \%$, is obtained for low resonance mode number, $s_{0}$. Consider the case $s_{0}=18$ : it implies $E_{\mathrm{sat}} \simeq 156 \mathrm{MV} / \mathrm{m}, R=0.249 \mathrm{~cm}, \eta^{(\max )}=94 \%, N^{(\mathrm{tl}),(\mathrm{opt})}=$ $10^{6}$. Figure 10 shows the wake dynamics for this specific example. The trailing bunch is located between $7.5 \mathrm{~cm}$ and $9.5 \mathrm{~cm}$ behind the trigger bunch (schematically represented by the dashed black line); the number of microbunches is 2000. As predicted by our analytical formulations the wakefield behind the e-beam is reduced to almost zero, which is indicative of high efficiency. However, since the wakefield value is not uniform along the train it is expected in practice that the electrons in the train will have a substantial energy spread. Such acceleration configuration may be useful for medical application where high efficiency is crucial and large energy spread can be tolerable.

A broader perspective of the high efficiency regime is revealed in Fig. 11 where this maximum efficiency (solid curve) for pointlike microbunches and $M^{(\mathrm{tl})}=2000$ as a function of the resonance mode number, $s_{0}$, is illustrated. Clearly, maximum efficiency is achieved at small values of $s_{0}$. Also, the corresponding optimal number of electrons per microbunch, $N^{(\mathrm{tl}),(\mathrm{opt})}$, [Eq. (D15)] is of the order of a few millions at small values of resonance number according

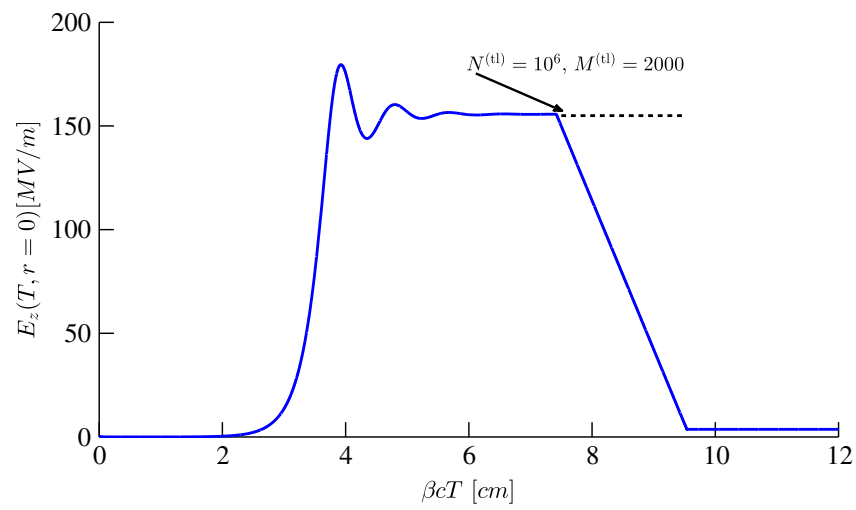

FIG. 10. Wake dynamics (solid curve) for resonance mode number of $s_{0}=18$. The second trailing train bunch (dashed curve) is located at the saturation regime.

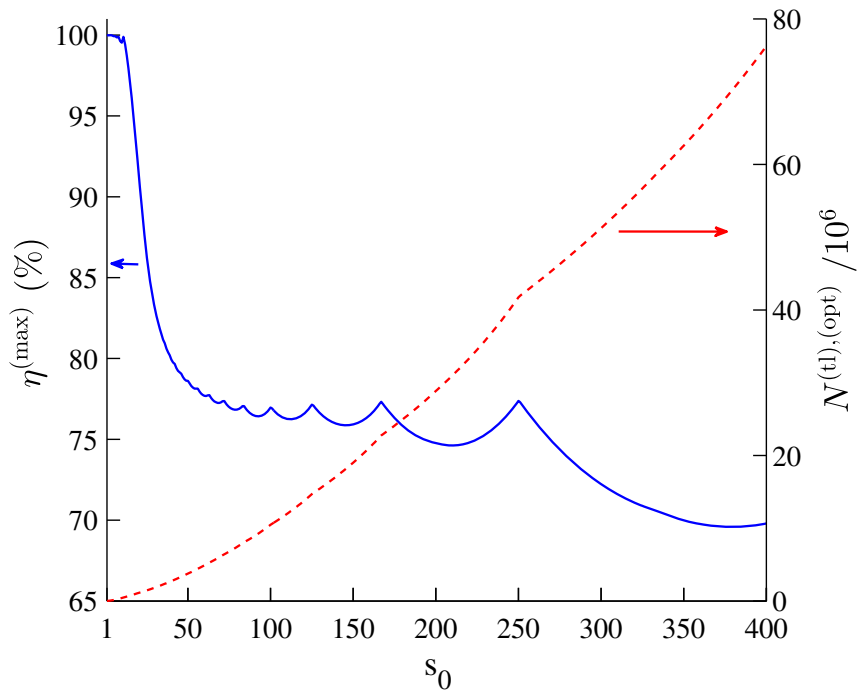

FIG. 11. The maximum efficiency (blue solid curve) and the corresponding optimum electrons per microbunch, $N^{(\mathrm{tl}) \text {, (opt) }}$, (red dashed curve) as function of the resonance mode $s_{0}$; the number of microbunches is 2000 .

to the dashed-curve of Fig. 11. However, since the value of saturated wake is proportional to $\sqrt{s_{0}}$, we realize the limitations: low $s_{0}$ facilitates high efficiency but the gradient is low (saturation) as well as the low optimal charge and vice versa, high $s_{0}$ leads to low efficiency but the gradient is high and so is the optimal charge. This is an additional important conclusion of the current study.

To complete the description of the acceleration process in this regime, the four frames of Fig. 12 show the energy
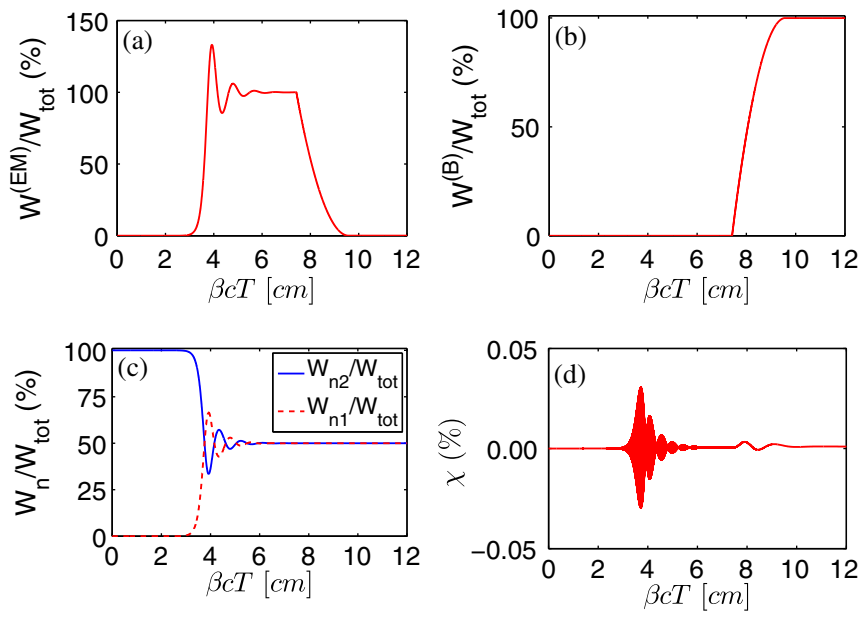

FIG. 12. Energy conservation of the example shown in Fig. 10. Specifically, Fig. 12 shows percentage of initial stored energy transferred to the electromagnetic energy [Fig. 12(a)], electron bunch [Fig. 12(b)], and Fig. 12(c) shows percentage of initial stored energy remaining in the AM. The fraction of stored energy in the upper population state level of the $\mathrm{AM}$ is $W_{\mathrm{n} 2} / W_{\text {tot }}=(1+\bar{n}) / 2$ and in the lower state level is $W_{\mathrm{n} 1} / W_{\text {tot }}=(1-\bar{n}) / 2$. Finally, Fig. 12(d) shows the numerical error in energy conservation. 
variation along the interaction region: the top left frame (a) reveals the normalized electromagnetic energy stored in the Cerenkov wake, top-right frame (b) illustrates the energy transferred to the trailing bunch, bottom-left frame (c) shows the energy in the upper (blue-solid-line) and the lower (red-dotted-line) energy state of the medium. Finally, in the bottom-right frame (d) the numerical error in energy conservation is revealed. In the interval of $0 \leq \beta c T \leq 4 \mathrm{~cm}$ the wake [Fig. 12(a)] is amplified in the linear regime and the PID is virtually constant such that most of the energy is deposited in the upper population state level. In the nonlinear regime ( $4 \mathrm{~cm} \leq \beta c T \leq 6 \mathrm{~cm}$ ), the upper energy-state is reduced and lower state is elevated which result in reduction of the PID energy. The total energy of the wake and the PID in this region experience the Rabbi oscillation till the energy of the AM is completely depleted and 100\% of the initially stored energy in the AM is eventually transferred to the wake. At this point we inject the second trailing bunch that fills the interval $7 \mathrm{~cm} \leq \beta c T \leq 9 \mathrm{~cm}$. In this region it is confirmed that the wake energy delivered to the trailing bunch [Fig. 12(c)] is close to $100 \%$ (in the simulation it is 99\%). The maximum deviation from energy conservation shown in Fig. 12(d) is less than $0.05 \%$.

\section{B. High-energy acceleration with low-efficiency}

Another regime of interest is that corresponding to high-energy physics applications where the beam quality is of major importance and efficiency is a secondary consideration. In this case it is necessary to have a highgradient with smallest possible energy-spread and in what follows we present the necessary trade-off in the design parameters. Consider $N^{(\mathrm{tl})}=10^{6}, M^{(\mathrm{tl})}=2000$, and $s_{0}=$ 360 in which case the saturated wake is $E_{\mathrm{sat}}=0.7 \mathrm{GV} / \mathrm{m}$, the efficiency is $\eta=2.1 \%$, and the energy gain after $3 \mathrm{~m}$ is $\Delta E_{k} \approx 2 \mathrm{GeV}$. We confirmed by numerical simulations (not shown here) that the gradient along the trailing train of bunches is uniform within less than $1 \%$.

\section{Constant-gradient acceleration in the linear regime}

Another approach to accelerate electrons with constant wake amplitude has been proposed in Ref. [15]. Its essence is that in the linear regime, the exponential growth of the wake compensates exactly for the beam loading.

According to Ref. [15] the condition for constant gradient in terms of our parameter notations with single trigger microbunch $\left(M^{(\mathrm{tg})}=1\right)$ reads

$$
N^{(\mathrm{tl})}=N^{(\mathrm{tg})} e^{\delta \omega_{\frac{\lambda}{\beta c}}^{\lambda_{0}}\left(N_{\text {space }}+\frac{1}{2}\right)}\left(1-e^{-\delta \omega \frac{\lambda_{0}}{\beta c}}\right)
$$

where $N_{\text {space }}$ is the integer number of wavelengths behind the trigger that the trailing bunch is located. As an example we consider $s_{0}=360$ with $N_{\text {space }}=4200$, the number of electrons in the trigger bunch is $N^{(\mathrm{tg})}=10^{4}$ and the spatial growth rate is $\delta \omega / \beta c=357 \mathrm{~m}^{-1}$. After substituting these parameters into Eq. (21) the required number of electrons per microbunch is $N^{(\mathrm{tl})}=303.4 \times 10^{6}$. In practice, since the PID varies slowly even in the quasilinear regime, the theoretical estimate of electrons per microbunch can be higher than from the numerical simulation. In addition, the number of microbunches in the trailing bunch should be small enough such that the effective growth rate in the location of the bunch will be virtually uniform. Thus, we choose $M^{(\mathrm{t})}=50$ and for an initial guess we started with $N^{(\mathrm{tl})}=303.4 \times 10^{6}$. Simulations indicate (Fig. 13) that for $N^{(\mathrm{tl})}=150 \times 10^{6}$ nearly uniform gradient of $E_{z}=$ $137 \mathrm{MV} / \mathrm{m}$ with maximum deviation of $0.44 \%$ and the resulting efficiency is $0.6 \%$. Consequently, for a relatively low but constant accelerating gradient it is possible to accelerate larger number of electrons per microbunch than in the previous approach.

\section{Emittance}

The normalized emittance is an important parameter which describes how well the beam is confined in space and momentum. For relativistic beam the normalized emittance is given by $\varepsilon=\gamma k_{\beta} \sigma^{2}$ where $\sigma^{2}$ is the transverse spot size of the beam and $k_{\beta}$ is the betatron wave number which is proportional to the radial focusing force. A necessary condition to preserve the emittance is beam matching. In a plasma-based accelerator the emittance can grow due to finite energy spread of the beam since the focusing force depends on the radius [16]. In addition, when considering staging, the emittance can grow due to finite bunch length since the focusing force depends on the plasma density [17]. Nevertheless, emittance growth due to the finite bunch

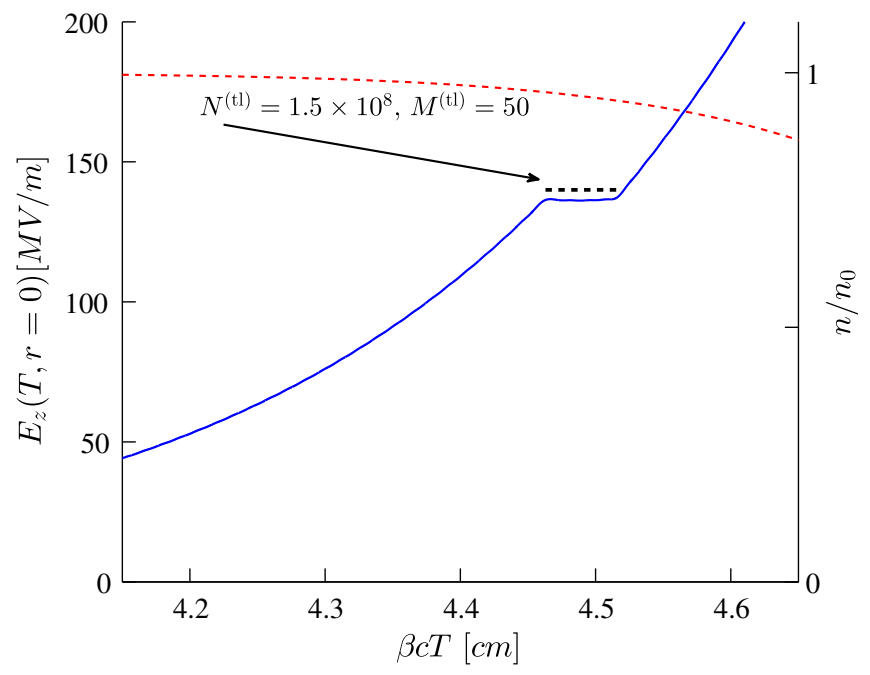

FIG. 13. Wake dynamics (solid curve) for resonance mode number of $s_{0}=360$ in the length interval of $4.15 \mathrm{~cm} \leq$ $\beta c T \leq 4.65 \mathrm{~cm}$. The second trailing train bunch (dash-dotted curve) is located at the quasilinear regime, where the normalized PID (dashed curve), $n / n_{0}$, is nearly constant and approximately equals to one. 
length can be suppressed if the beam parameters are matched to the betatron frequency $[17,18]$.

Another mechanism that can contribute to the growth of the emittance is the Coulomb scattering in the plasma [19-21]. In a plasma-based accelerator the growth of the emittance due to beam ion channel scattering and beam vapor scattering is (see Appendix E for more details)

$$
\Delta \varepsilon_{\mathrm{PWFA}}=\sqrt{2} r_{e} S\left(\sqrt{\gamma_{f}}-\sqrt{\gamma_{i}}\right),
$$

where $r_{e}$ is the classical electron radius, $m_{e} c^{2}\left(\gamma_{i}-1\right)$ is the initial beam energy and $m_{e} c^{2}\left(\gamma_{f}-1\right)$ is the final beam energy. In addition, $S$ is a parameter defined in Appendix $\mathrm{E}$ which depends on the ion channel radius, $R_{b}$, the atomic radius, $R_{a}$, and the atomic number of the vapor, $Z$. As an example, emittance growth from doubling the beam energy from $500 \mathrm{GeV}$ is of the order of $\Delta \varepsilon_{\mathrm{PWFA}} \approx$ $10^{-2} \mathrm{~mm}$-mrad assuming $Z=60, R_{a}=10^{-10} \mathrm{~m}$, and $R_{b}=2.5 \times 10^{-5} \mathrm{~m} \mathrm{[20].}$

In our structure, the emittance can grow due to the scattering of the beam through a neutral gas mixture that consists of an AM. More specifically, the growth of the emittance is (see Appendix E for more details)

$$
\begin{aligned}
\Delta \varepsilon_{\mathrm{AM}-\mathrm{TBA}}= & \frac{1}{2}\left(\frac{14 \mathrm{MeV}}{m_{e} c^{2}}\right)^{2} \frac{\rho}{X_{0}}\left(\frac{m_{e} c^{2} / e}{\left|E_{\mathrm{sat}}\right|}\right)^{1.5} \\
& \times \frac{1}{\sqrt{(\omega / c) \sqrt{\bar{\varepsilon}}}}\left(\sqrt{\gamma_{f}}-\sqrt{\gamma_{i}}\right),
\end{aligned}
$$

where $\rho$ is the weight density measured in $\mathrm{g} / \mathrm{cm}^{3}$ and $X_{0}$ is the radiation length measured in $\mathrm{g} / \mathrm{cm}^{2}$. In our structure, the elevation of the emittance can be suppressed at low gas density, high accelerating gradient, high resonance frequency, and high Cerenkov parameter. For a given active gas mixture the resonance frequency is constant and in order that the saturation length will be as small as possible the Cerenkov parameter cannot be increased significantly. In addition, to avoid field ionization the value of the saturated wake cannot exceed significantly gradient of $\mathrm{GV} / \mathrm{m}$. Hence, the main parameter that can minimize the emittance growth is the weight density which depends on the pressure, namely assuming ideal gas behavior $\rho=p \times$ $10^{5} / \tilde{R} T_{k}$, where $p$ is the pressure in atm, $\tilde{R}$ is the specific gas constant measured in $\mathrm{J} /(\mathrm{kg} \times K)$, and $T_{k}$ is the gas temperature measured in Kelvin.

For example, in a gas mixture of $\mathrm{CO}_{2}, \mathrm{~N}_{2}$, and $\mathrm{He}$ with ratio of $1: 1: 14[22,23]$ the specific gas constant is $\tilde{R}=$ $(1 / 16)\left(R_{\mathrm{CO}_{2}}+R_{\mathrm{N}_{2}}\right)+(14 / 16) R_{\mathrm{He}}=0.0625(189+297)+$ $0.875 \times 2000=1780 \mathrm{~J} /(\mathrm{kg} \times \mathrm{K})$ and the radiation length is $\quad X_{0}=\left[(1 / 16) / X_{\mathrm{CO}_{2}}+(1 / 16) / X_{\mathrm{N}_{2}}+(14 / 16) / X_{\mathrm{He}}\right]^{-1}=$ $(0.0625 / 36+0.0625 / 38+0.875 / 94)^{-1}=78.8 \mathrm{~g} / \mathrm{cm}^{2}$ [24]. Hence, from ideal gas law the weight density at room temperature $\left(T_{k}=300 \mathrm{~K}\right)$ and at pressure of $10 \mathrm{~atm}$ is
$1.872 \mathrm{~g} / \mathrm{cm}^{3}$. Assuming resonance mode number $s_{0}=360$ the wake at saturation is $E_{\text {sat }}=0.7 \mathrm{GV} / \mathrm{m}$. Thus, if the initial trailing train has $\gamma_{i}=600$, the emittance growth from doubling the energy of the trailing train is $\Delta \varepsilon_{\mathrm{AM}-\mathrm{TBA}} \approx$ $10 \mathrm{~mm}$-mrad. If we reduce the pressure by ten times, the Cerenkov parameter drops similarly [25] and also the weight density of the gas mixture. Keeping the same value of the wake amplitude at saturation, one obtains that the emittance growth from doubling the energy of the trailing train is $\Delta \varepsilon_{\text {AM-TBA }} \approx 1 \mathrm{~mm}$-mrad. According to Ref. [26] reducing the pressure from $10 \mathrm{~atm}$ to $1 \mathrm{~atm}$ will weakly modify the growth rate of the amplified wake, $\delta \omega$. Hence, for $\mathrm{CO}_{2}$ gas mixture with pressure of $1 \mathrm{~atm}$ the saturation length will be nearly the same. However, to keep the same saturation value, the waveguide radius should be increased by a factor of $(\sqrt{10} \approx 3$ ), namely the waveguide radius should be $15 \mathrm{~cm}$.

\section{SUMMARY}

In this study we investigated the wake dynamics and the efficiency of an AM two-beam accelerator (AM-TBA) driven by a realistic (discrete) distribution of electrons using both analytic and numerical methods. In what follows we summarize our main results and conclusions.

\section{A. Wake dynamics}

In the first part of this study we have studied the wake dynamics for a trigger bunch consisting of a finite number of electrons. In principle, it was shown that even a single electron in the trigger bunch can generate wakefield that will reach saturation within a few $\mathrm{cm}$ 's. However, since in practice the initial location of the electron is random, the phase of the wake will have a different value in each simulation which is impractical for acceleration of a second trailing bunch. In case of multiple electrons we distinguish between two regimes: if the number of electrons is below a threshold value $\left(N^{(\mathrm{tg})}<N_{\text {thr }}^{(\text {act })}\right)$ the amplitude is set by the saturation value but the phase is still random again, irrelevant for acceleration. However, if this value is exceeded $\left(N^{(\mathrm{tg})}>N_{\text {thr }}^{(\mathrm{act})}\right)$ the wake's phase-spread diminishes and values which make the paradigm relevant for acceleration of electrons, were demonstrated.

We argue that this behavior differs from self-amplified spontaneous emission (SASE) in lasers as photons set a constraint on the frequency of the field, whereas electrons set a constraint of the phase velocity of the field. Hence, the wake that is generated from a number of electrons larger than a threshold value has a small jitter in amplitude and phase. This wake that its rise time is of the order of the electrons' spread length over the speed of light can be employed as a seed pulse for the backward Raman amplification in plasma [27].

Another positive effect of a large number of electrons in the trigger bunch is reduced saturation length. While clearly below the threshold value operation is unacceptable, 
increasing the number of electrons to too high values is detrimental since significant energy is coupled to adjacent Bessel modes and multi-mode operation is unacceptable from the standpoint of the trailing electrons.

\section{B. Energy conversion efficiency}

In the second part of this study the energy conversion efficiency including beam loading has been considered. Specifically, we inject in addition to the trigger bunch a second trailing bunch that can be accelerated by the amplified wake. Since the trailing bunch generates a wake that tends to reduce the amplified wake, it is not possible to have both high energy efficiency and constant gradient. Hence, we propose three different alternatives (see Table II) to obtain either high energy efficiency but with relatively high energy spread or high energy gain but with low conversion efficiency. In the first two configurations the accelerating trailing bunch is located in the region the amplified wake reaches saturation and in the third configuration the bunch is located in the quasilinear region.

For the case used to illustrate the potential of our paradigm, a $\mathrm{CO}_{2}$ gas mixture, efficiency exceeding $90 \%$ in the saturation region can be obtained if the radius is designed such that the resonant mode is less than 20 and a maximum wake amplitude (at saturation) of the order of hundreds $\mathrm{MV} / \mathrm{m}$. Specifically, wall's radius $R=0.249 \mathrm{~cm}$, resonant mode $s_{0}=18$, the number of electrons per microbunch of $N^{(\mathrm{tl})}=10^{6}$ and number of microbunches of $M^{(\mathrm{tl})}=2000$, the maximum gradient is $E_{\text {sat }}=156 \mathrm{MV} / \mathrm{m}$, and the efficiency is $\eta=94 \%$. Since the gradient is reduced in the location of the trailing bunch, the energy spread is expected to be large. Such acceleration configuration may be useful for medical application where energy spread is not so crucial as the efficiency.

Nearly constant gradient can be obtained at higher resonance mode but with significantly lower efficiency. For example, in the second configuration the waveguide radius is increased to $R=5.065 \mathrm{~cm}$ in order to amplify the wake at resonance number $s_{0}=360$. As a result, the gradient at saturation is increased to $E_{\mathrm{sat}}=0.7 \mathrm{GV} / \mathrm{m}$. Keeping the same number of electrons and the same length of the trailing bunch as in the former configuration the efficiency is reduced significantly to $\eta=2.1 \%$. For structure length of $3 \mathrm{~m}$ the energy gain is $2 \mathrm{GeV}$.

Constant gradient with higher charge can be achieved in the quasilinear regime of the wake amplification. On the one hand the AM tends to increase the amplitude while on the other hand, we may design the loading such that it exactly compensates this increase, such that the net amplitude is constant. As an example, in the third configuration, we have shown that for $N^{(\mathrm{tl})}=150 \times 10^{6}$ and $M^{(\mathrm{tl})}=50$ the wake amplitude is constant and equal to $137 \mathrm{MV} / \mathrm{m}$. However, the efficiency is relatively low $(0.6 \%)$ compared to the previous configurations.

In Sec. VD the emittance growth from Coulomb scattering in an active gas mixture was analyzed. First we have noticed that the amplified wake can be employed for both accelerating and focusing the trailing train; thus, there is no need for a Focus-Drift-Defocus-Drift lattice. Though the focusing force, which is proportional to the wake amplitude at saturation, can reduce the emittance growth, it practically cannot be set to high value due to field ionization limitation. Hence, in our structure emittance growth strongly depends on the gas pressure. As an illustrative example, we considered $\mathrm{CO}_{2}$ gas mixture with

TABLE II. Simulation parameters of the three accelerating configurations. For both the trigger and the trailing bunches each microbunch is a pointlike charge $\left(\Delta z^{(\mathrm{tg})}=\Delta z^{(\mathrm{tl})}=0\right)$. In addition, the trigger bunch consists of a single microbunch $\left(M^{(\mathrm{tg})}=1\right)$.

\begin{tabular}{|c|c|c|c|c|}
\hline Parameter & Designation & High efficiency & High energy gain & Constant gradient acceleration \\
\hline Medium resonance wavelength $\left(\mathrm{CO}_{2}\right)$ & $\lambda_{0}$ & $10.6 \mu \mathrm{m}$ & $10.6 \mu \mathrm{m}$ & $10.6 \mu \mathrm{m}$ \\
\hline Medium resonance bandwidth (at $10 \mathrm{~atm}$ ) & $\Delta \omega$ & $2.32 \times 10^{11} \mathrm{rad} / \mathrm{sec}$ & $2.32 \times 10^{11} \mathrm{rad} / \mathrm{sec}$ & $2.32 \times 10^{11} \mathrm{rad} / \mathrm{sec}$ \\
\hline Medium relative permittivity (at 10 atm) & $\epsilon_{r}$ & 1.00142 & 1.00142 & 1.00142 \\
\hline Medium growth rate coefficient & $\alpha$ & $1 \mathrm{~m}^{-1}$ & $1 \mathrm{~m}^{-1}$ & $1 \mathrm{~m}^{-1}$ \\
\hline e-beam Lorentz factor & $\gamma$ & 600 & 600 & 600 \\
\hline e-beam radius & $R_{\mathrm{b}}$ & $30 \mu \mathrm{m}$ & $30 \mu \mathrm{m}$ & $30 \mu \mathrm{m}$ \\
\hline $\begin{array}{l}\text { Trigger bunch-number of electrons } \\
\text { per microbunch }\end{array}$ & $N^{(\operatorname{tg})}$ & $10^{4}$ & $10^{4}$ & $10^{4}$ \\
\hline Waveguide radius & $R$ & $0.249 \mathrm{~cm}$ & $5.065 \mathrm{~cm}$ & $5.065 \mathrm{~cm}$ \\
\hline Resonance number & $s_{0}$ & 18 & 360 & 360 \\
\hline $\begin{array}{l}\text { Trailing bunch-number of electrons } \\
\text { per microbunch }\end{array}$ & $N^{(\mathrm{tl})}$ & $10^{6}$ & $10^{6}$ & $150 \times 10^{6}$ \\
\hline Trailing bunch-number of microbunches & $M^{(\mathrm{tl})}$ & 2000 & 2000 & 50 \\
\hline $\begin{array}{l}\text { The distance between the trailing and } \\
\text { the trigger bunches }\end{array}$ & $\Delta L$ & $7 \mathrm{~cm}$ & $8.5 \mathrm{~cm}$ & $4.2 \mathrm{~cm}$ \\
\hline $\begin{array}{l}\text { Maximum wake amplitude in the } \\
\text { trailing bunch location }\end{array}$ & $E_{\max }^{(\mathrm{tl})}$ & $156 \mathrm{MV} / \mathrm{m}$ & $0.7 \mathrm{GV} / \mathrm{m}$ & $137 \mathrm{MV} / \mathrm{m}$ \\
\hline Efficiency & $\eta$ & $94 \%$ & $2.1 \%$ & $0.6 \%$ \\
\hline
\end{tabular}


$E_{\text {sat }}=0.7 \mathrm{GV} / \mathrm{m}$ and trailing beam which doubles its energy where its initial energy is $300 \mathrm{MeV}(\gamma=600)$. For pressure of $10 \mathrm{~atm}$ the estimated emittance growth is $10 \mathrm{~mm}$-mrad and when the pressure is reduced to $1 \mathrm{~atm}$ the emittance growth is decreased to $1 \mathrm{~mm}$-mrad. At low pressure of $1 \mathrm{~atm}$ the main parameter that is changed is the waveguide radius which increased to $15 \mathrm{~cm}$. Comparing to PWFA the typical growth of emittance from Coulomb scattering is of the order of $10^{-2} \mathrm{~mm}$-mrad for doubling the beam energy from $500 \mathrm{GeV}\left(\gamma=10^{6}\right)$.

\section{Proof of principle experiment}

At this point we would like to suggest a proof of principle experiment of Cerenkov wake amplification with enhanced growth rate. If we use the same setup of the PASER experiment [11] but with pressure of 0.6 atm rather than $0.25 \mathrm{~atm}$, then the $45 \mathrm{MeV}$ modulated train of microbunches will emit Cerenkov wake that will be amplified. Under this pressure with gas mixture of $\mathrm{CO}_{2}, \mathrm{~N}_{2}$, and $\mathrm{He}$ with ratio of $2: 2: 3$, the Cerenkov slippage parameter is $\bar{\epsilon}=1.32 \times 10^{-4}[15]$ and the spatial growth of the amplified Cerenkov wake is projected to be $\delta \omega / c=407.61 / \mathrm{m}$. In this experiment we can compare between laser pulse amplification in the active $\mathrm{CO}_{2}$ gas mixture and Cerenkov wake amplification in which the latter is expected to be significantly larger due to the enhanced growth rate.

\section{ACKNOWLEDGMENTS}

This work was supported by the Bi-National Science Foundation (BSF) and by the Israel Science Foundation (ISF).

\section{APPENDIX A: WAKEFIELD IN A PASSIVE MEDIUM}

In this appendix we calculate the wakefield of an electron train bunch that propagates in a passive medium $(\bar{n}=0)$. Consider train of $M^{(\mathrm{tg})}$ microbunches with one resonance wavelength period. In every experiment each microbunch consists of $N^{(\mathrm{tg})}$ electrons that initially are randomly distributed over a length of $\Delta z^{(\mathrm{tg})}$ corresponding to initial spread of the electrons phase at the resonance mode $s_{0}$ to be $2 \psi=\omega_{0} \Delta z^{(\operatorname{tg})} /(\beta c)$. Note that the superscript (tg) indicates that the parameter describes the trigger bunch. In a given experiment, the envelope of the longitudinal wake at the resonance mode, $s_{0}$, [defined in Eq. (9)] is

$$
E_{z, d}^{(\mathrm{pas})}=\frac{1}{N^{(\mathrm{tg})} M^{(\mathrm{tg})}} \sum_{\nu=1}^{M^{(\mathrm{tg})}} \sum_{m=1}^{N^{(\mathrm{g})}} E_{\nu, m, d}^{(\mathrm{pas})},
$$

where the index $d\left(d=1,2, \ldots N^{(\exp )}\right)$ represents the experiment number and $N^{(\exp )}$ is the number of experiments or in our study the number of the numerical simulations. Also,

$$
E_{\nu, m, d}^{(\mathrm{pas})}=E_{s_{0}} e^{i\left[\Gamma_{m, d}+2 \pi(\nu-1)\right]} \Theta\left(T+\frac{z_{m, d}^{(\mathrm{tg})}}{\beta c}\right),
$$

$E_{s_{0}}=e N^{(\mathrm{tg})} M^{(\mathrm{tg})} J_{c}\left(\chi_{s_{0}}\right) /\left[\epsilon_{0} \epsilon_{r} \pi R^{2} J_{1}^{2}\left(p_{s_{0}}\right)\right]$ is the wakefield amplitude for a pointlike bunch and $J_{c}(x) \equiv$ $2 J_{1}(x) / x$. In addition, $z_{m, d}^{(\mathrm{tg})}\left(m=1,2, \ldots N^{(\mathrm{tg})}\right)$ is the initial electron location with a spread of $\Delta z^{(\mathrm{tg})} \equiv$ $\max \left(z_{m}^{(\mathrm{tg})}\right)-\min \left(z_{m}^{(\mathrm{tg})}\right), \Gamma_{m, d}=\omega_{0} z_{m, d}^{(\mathrm{tg})} /(\beta c)$ is the electron phase at the resonance mode, and $\Theta(\cdot)$ is the Heaviside step function. In each experiment the random variable $\Gamma_{m, d}$ is uniformly distributed in the interval $(-\psi, \psi)$. Thus, in the limit of $T>\max -\left[z_{m}^{(\mathrm{tg})} /(\beta c)\right]$ the averaged wake is

$$
\begin{aligned}
\left\langle E_{z}^{(\mathrm{pas})}\right\rangle & \equiv \lim _{N^{(\exp )} \rightarrow \infty} \frac{1}{N^{(\exp )}} \sum_{d=1}^{N^{(\text {exp })}} E_{z, d}^{(\mathrm{pas})} \\
& =E_{S_{0}} \frac{1}{2 \psi} \int_{-\psi}^{\psi} d \Gamma e^{i \Gamma} .
\end{aligned}
$$

Hence, the averaged wake (over a large number of independent experiments) in a passive medium for $T>$ $\max \left[z_{m}^{(\mathrm{tg})} /(\beta c)\right]$ is

$$
\left\langle E_{z}^{(\mathrm{pas})}\right\rangle=E_{s_{0}} \operatorname{sinc}(\psi),
$$

where $\operatorname{sinc}(x) \equiv \sin (x) / x$. Now, we estimate the jitter of the wake by calculating its standard deviation. First, the squared absolute value of the wake is calculated by using Eq. (A1)

$$
\begin{aligned}
\left|E_{z, d}^{(\mathrm{pas})}\right|^{2}= & \left(\frac{E_{s_{0}}}{N^{(\mathrm{g})}}\right)^{2} \\
& \times\left[\sum_{m=m^{\prime}} 1+\sum_{m \neq m^{\prime}} e^{i\left(\Gamma_{m, d}-\Gamma_{m^{\prime}, d}\right)}\right] .
\end{aligned}
$$

Using Eq. (A5) the averaged squared absolute value of the wake is

$$
\begin{aligned}
\left\langle\left|E_{z}^{(\mathrm{pas})}\right|^{2}\right\rangle= & \left(\frac{E_{S_{0}}}{N^{(\mathrm{tg})}}\right)^{2}\left\{N^{(\mathrm{tg})}\right. \\
& \left.+\left[\left(N^{(\mathrm{tg})}\right)^{2}-N^{(\mathrm{tg})}\right] \operatorname{sinc}^{2}(\psi)\right\} .
\end{aligned}
$$

Note that the first term in Eq. (A6) represents the noncoherent (spontaneous) power contribution whereas the second term reveals the coherent power counterpart. Using Eqs. (A4) and (A6) the standard deviation is

$$
\begin{aligned}
\Delta E_{z}^{(\mathrm{pas})} & \equiv \sqrt{\left\langle\left|E_{z}^{(\mathrm{pas})}\right|^{2}\right\rangle-\left|\left\langle E_{z}^{(\mathrm{pas})}\right\rangle\right|^{2}} \\
& =\frac{\left|E_{S_{0}}\right| \sqrt{1-\operatorname{sinc}^{2}(\psi)}}{\sqrt{N^{(\mathrm{tg})}}} .
\end{aligned}
$$


Hence, the wake jitter is estimated by

$$
\frac{\Delta E_{z}^{(\mathrm{pas})}}{\left|\left\langle E_{z}^{(\mathrm{pas})}\right\rangle\right|}=\frac{\sqrt{1-\operatorname{sinc}^{2}(\psi)}}{\sqrt{N^{(\mathrm{tg})}}|\operatorname{sinc}(\psi)|} .
$$

\section{APPENDIX B: WAKEFIELD IN AN ACTIVE MEDIUM}

In this appendix we repeat the calculation from the above for a wake propagating in an AM. As in the previous appendix of passive medium the train bunch consists of $M^{(\mathrm{tg})}$ microbunches with $N^{(\mathrm{tg})}$ electrons in each microbunch.

The linear response of the wakefield $(\bar{n}=1)$ at resonance mode, $s_{0}$, generated from such trigger bunch is

$$
\begin{aligned}
E_{z, d}^{(\mathrm{act})}= & \frac{e^{\delta \omega T}}{N^{(\mathrm{tg})} M^{(\mathrm{tg})}} \sum_{\nu=1}^{M^{(\mathrm{tg})}} \sum_{m=1}^{N^{(\mathrm{gg})}} \bar{E}_{\nu, m, d}^{(\mathrm{pas})} \\
& \times \frac{1}{2}\left(1+\frac{1}{1+4 q}\right) e^{u \Gamma_{m, d}+u[2 \pi(\nu-1)]},
\end{aligned}
$$

where $\delta \omega$ is the linear growth rate defined in Eq. (11), $u=\delta \omega / \omega_{0}$, and $q=\delta \omega / \Delta \omega$.

In addition, without significant loss of generality it is assumed that $\Delta \omega \ll \omega_{0}, \quad \delta \omega / \omega_{0} \ll 1, \quad$ and $T>\max \left[z_{m} /(\beta c)\right]$. Note that in the limit that growth rate tends to zero $(\delta \omega \rightarrow 0)$ we obtain that $q \rightarrow 0$ and $u \rightarrow 0$. Hence, Eq. (B1) degenerates to Eq. (A1) as expected since in this case the medium becomes passive.

In the limit of large number of experiments the averaged wake becomes

$$
\left\langle E_{z}^{(\mathrm{act})}\right\rangle=E_{s_{0}} e^{\delta \omega T} G F
$$

where

$$
\begin{aligned}
G\left(q, u, M^{(\mathrm{tg})}\right) \equiv & \frac{1}{2}\left(1+\frac{1}{1+4 q}\right) \\
& \times e^{\pi u\left(M^{(\mathrm{tg})}-1\right)} \frac{\sinh \left(\pi u M^{(\mathrm{tg})}\right)}{M^{(\mathrm{tg})} \sinh (\pi u)}
\end{aligned}
$$

and

$$
\begin{aligned}
F(u, \psi) & \equiv \operatorname{sinc}(\psi-i u) \\
& =\frac{\sin (\psi) \cosh (\psi u)-i \cos (\psi) \sinh (\psi u)}{\psi(1-i u)} .
\end{aligned}
$$

For $u \ll 1$ which is the case of most of the active media, $M^{(\mathrm{tg})} u \ll 1$, and $q \gg 1$ the wake is

$$
\left\langle E_{z}^{(\text {act })}\right\rangle \simeq \frac{1}{2} E_{S_{0}} e^{\delta \omega T}[\operatorname{sinc}(\psi)-i u \cos (\psi)] .
$$

In this regime there are two important limits of $\psi \ll 1$ and $\psi \approx \pi$. In the first limit of small initial spread the wake is

$$
\left\langle E_{z}^{(\mathrm{act})}\right\rangle \simeq \frac{1}{2} E_{S_{0}} e^{\delta \omega T} .
$$

However, in the second limit of $\psi \simeq \pi$ the wake is

$$
\left\langle E_{z}^{(\mathrm{act})}\right\rangle \simeq \frac{1}{2} E_{S_{0}} e^{\delta \omega T} i u
$$

This means that the wake at $\psi \simeq \pi$ is smaller by a factor of $1 / u$ than the wake at $\psi \ll 1$. In the limit of $M^{(\mathrm{tg})} u \gg 1$ the wake is similar as in Eq. (B5) but is multiplied by the factor $e^{2 \pi u M^{(\mathrm{gg})}} / M^{(\mathrm{tg})}$.

Similarly as in the previous appendix, we calculate $\left|E_{z}^{\text {(act) }}\right|^{2}$ to estimate the jitter of the wake. Thus, from Eq. (B1) we have

$$
\begin{aligned}
\left|E_{z, d}^{(\mathrm{act})}\right|^{2}= & \frac{\left|E_{s_{0}}\right|^{2} e^{2 \delta \omega T}}{\left(N^{(\mathrm{tg})} M^{(\mathrm{tg})}\right)^{2}} \frac{1}{4}\left(1+\frac{1}{1+4 q}\right)^{2} \\
& \times \sum_{\nu, m, \nu^{\prime}, m^{\prime}} e^{i \Gamma_{m, d}} e^{u \Gamma_{m, d}+u[2 \pi(\nu-1)]} \\
& \times e^{-i \Gamma_{m^{\prime}, d}} e^{u \Gamma_{m^{\prime}, d}+u\left[2 \pi\left(\nu^{\prime}-1\right)\right]} .
\end{aligned}
$$

Hence, in the limit of $N^{(\exp )} \gg 1$ and $M^{(\mathrm{tg})} \gg 1$ we obtain

$$
\begin{aligned}
\left\langle\left|E_{z, s_{0}}^{(\mathrm{act})}\right|^{2}\right\rangle= & e^{2 \delta \omega T}\left(\frac{\left|E_{s_{0}}\right|}{N^{(\mathrm{tg})}}\right)^{2} G^{2}\left\{N^{(\mathrm{tg})} \operatorname{sinc}(2 u \psi)\right. \\
& +\left[\left(N^{(\mathrm{tg})}\right)^{2}-N^{(\mathrm{tg})}\right] \\
& \left.\times|\operatorname{sinc}(\psi-i u)|^{2}\right\}
\end{aligned}
$$

As in the previous appendix, the first term in Eq. (B9) represents the noncoherent (spontaneous) contribution whereas the second term reveals the coherent counterpart.

For $u \psi \ll 1$ the standard deviation is

$$
\begin{aligned}
\Delta E_{z}^{(\mathrm{act})} & \equiv \sqrt{\left\langle\left|E_{z}^{(\mathrm{act})}\right|^{2}\right\rangle-\left|\left\langle E_{z}^{(\mathrm{act})}\right\rangle\right|^{2}} \\
& \simeq \frac{\left|\left\langle E_{z}^{\text {(act) }}\right\rangle\right| \sqrt{1-|F|^{2}}}{\sqrt{N^{(\mathrm{tg})}}|F|} .
\end{aligned}
$$

The ratio between the standard deviation to average value is

$$
\frac{\Delta E_{z}^{(\text {act })}}{\left|\left\langle E_{z}^{(\text {act })}\right\rangle\right|}=\frac{\sqrt{1-|F|^{2}}}{\sqrt{N^{(\mathrm{tg})}|F|}} .
$$

Thus, for $N^{(\mathrm{tg})} \geq N_{\mathrm{thr}}^{(\mathrm{act})}$, where $N_{\mathrm{thr}}^{(\mathrm{act})} \equiv\left(1-|F|^{2}\right) /|F|^{2}$ the coherent part of the wake is larger than the noncoherent counterpart [see Eq. (B9)]. For $\psi \ll 1$ the threshold of electron number in the microbunch is $N_{\text {thr }}^{\text {(act) }}=0$. This is an expected result since coherent wake without jitter is 
obtained in the limit of a point-charge like microbunch. However, for $\psi \simeq \pi$ the threshold is $N_{\mathrm{thr}}^{\text {(act) }}=1 / u^{2}$. Comparing to the passive medium case presented in the previous section the threshold of electron number is similar as in the AM case up to a factor of $|F(u, \psi)|^{2}$. Interestingly, while in the passive medium case for initial spread of $2 \psi=2 \pi$ the electron number threshold tends to infinity, in the AM case the threshold tends to a finite number of $1 / u^{2}$. Note that in the limit of passive medium $(u=0)$ one obtains that $|F(u=0, \psi)|^{2}=1$ and the threshold is the same in both cases of passive and active media.

In case of normal electron distribution rather than uniform distribution the whole previous theoretical results are the same except that the function $F(u, \psi)$ is changed. More specifically, $F^{(\mathrm{N})}(u, \psi)=\exp \left[-\sigma^{2}\left(1-u^{2}\right) / 2+i \sigma u\right] \simeq$ $\exp \left(-\sigma^{2} / 2+i \sigma u\right)$, where $\sigma=2 \psi / \sqrt{12}$ is the standard deviation of the normal distribution. Note that the factor $\sqrt{12}$ in the standard deviation comes from the demand that both the uniform and the normal distributions have the same variance. In addition, for $\psi \ll 1$ the threshold of electron number in both the normal and uniform electron distributions is zero.

\section{APPENDIX C: SATURATION LENGTH}

An important parameter in our AM-TBA is the saturation length, which is the distance behind the trigger bunch that the amplified wake reaches saturation.

To obtain an explicit expression for the saturation length or time, the amplified wakefield behind the trigger bunch is needed to be calculated from the previous appendix. Specifically, in the limit of $N^{(\mathrm{tg})} \gg 1, M^{(\mathrm{tg})} \delta \omega / \omega_{0} \ll 1$, and $\delta \omega / \Delta \omega \gg 1$ the absolute value of the wake just behind the trigger bunch that is uniformly distributed is [see Eq. (B5)]

$$
\begin{aligned}
\left|\left\langle E_{z, s_{0}}^{(\mathrm{act})}\right\rangle\right| \simeq & \frac{1}{2}\left|E_{s_{0}}\right| e^{\delta \omega T_{1}} \\
& \times \sqrt{u^{2} \cos ^{2}(\psi)+\operatorname{sinc}^{2} \psi}
\end{aligned}
$$

where $T_{1}=\Delta z /(2 \beta c)=\psi / \omega_{0}$. Substituting Eq. (C1) into Eq. (10) and assuming that $\delta \omega T_{1}=u \psi \ll 1$ will yield saturation time of

$$
t_{\text {sat }}=\frac{1}{\delta \omega} \ln \left(\sqrt{\frac{U_{\mathrm{a}}}{U_{0}}}\right)+\frac{20}{\Delta \omega},
$$

where the initial stored energy per length in the AM is

$$
U_{\mathrm{a}}=\frac{1}{2} \hbar \omega_{0} n_{0} \pi R^{2}
$$

and the associated initial energy per length of the trigger bunch is

$$
U_{0}=\frac{\left(e N^{(\mathrm{tg})} M^{(\mathrm{tg})}\right)^{2}}{32 \epsilon_{0} \epsilon_{r} \pi R^{2}} \frac{|F|^{2}}{J_{1}^{2}\left(p_{s_{0}}\right)} .
$$

Thus, the saturation length is $L_{\mathrm{sat}}=\beta c t_{\mathrm{sat}}$.

\section{APPENDIX D: BEAM LOADING}

In this Appendix we calculate the efficiency of the AMTBA for trailing bunch that is located at the saturation regime. Consider trailing bunch of the form of

$$
\begin{aligned}
J_{z}^{(\mathrm{tl})}= & -e \sum_{\nu=1}^{M^{(\mathrm{t})}} \sum_{m=1}^{N^{(\mathrm{t})}} \delta\left[T+\frac{z_{m}^{(\mathrm{tl})}+(\nu-1) \lambda_{0}}{\beta c}\right] \\
& \times \frac{\Theta\left(R_{b}-r\right)}{\pi R_{b}^{2}}
\end{aligned}
$$

where $T=t-z /(\beta c)$, the superscript (tl) indicates that the parameter describes the trailing bunch, $N^{(\mathrm{tl})}$ is the number of electron in each microbunch and $M^{(\mathrm{tl})}$ is the number of microbunches. In addition, for each microbunch $z_{m}^{(\mathrm{tl})}$ $\left(m=1,2, . . N^{(\mathrm{tl})}\right)$ is the initial electron location.

The trailing bunch propagates in the region of saturation where the AM is fully depleted or PID is zero. In this analysis it is assumed that the medium becomes a passive transparent medium with dielectric coefficient $\epsilon_{r}$. In the absence of loading the wake amplitude at saturation is

$$
E_{\mathrm{sat}}=\frac{1}{2}\left[\sqrt{\frac{\hbar \omega_{0} n_{0}}{\epsilon_{0} \epsilon_{r}}} \frac{J_{0}\left(k_{s_{0}} r\right)}{J_{1}\left(p_{s_{0}}\right)} e^{i \omega_{0} T}+\text { c.c. }\right],
$$

where the absolute value of the wake amplitude at saturation is determined by energy conservation [see Eq. (12)] and it is assumed that the initial phase-spread of the electrons in the trigger bunch at the resonance mode is small $(\psi \ll 1)$. Moreover, the number of electrons in the trigger bunch is sufficiently large to have small spread of the wake phase but small enough to avoid oscillations at saturation in the amplitude. The wake of the trailing bunch is

$$
E_{z}^{(\mathrm{ll})}=\frac{1}{2}\left[\sum_{s=1}^{\infty} E_{z, s}^{(\mathrm{ll})} J_{0}\left(k_{s} r\right) e^{i \omega_{s} T}+\text { c.c. }\right],
$$

where $\omega_{s}=k_{s} c /\left(\sqrt{\epsilon_{\mathrm{r}} \epsilon_{c}}\right)$ and the amplitude of the wake is

$$
E_{z, s}^{(\mathrm{tl})}=\sum_{\nu=1}^{M^{(\mathrm{t})}} \sum_{m=1}^{N^{(\mathrm{t})}} \frac{e}{\epsilon_{0} \epsilon_{r} \pi R^{2}} \frac{J_{c}\left(\chi_{s}\right)}{J_{1}^{2}\left(p_{s}\right)} e^{i_{\frac{\omega_{5} \lambda_{0}}{2 \pi c}} \Psi_{m, \nu}},
$$

where $\Psi_{m, \nu}=2 \pi\left[z_{m}-\lambda_{0}(\nu-1)\right] /\left(\beta \lambda_{0}\right)$ and $\chi_{s}=k_{s} R_{\mathrm{b}}$. Therefore, in the presence of the accelerating train bunch the total wakefield at the saturation is $E_{\mathrm{tot}}=E_{\mathrm{sat}}+E_{z}^{(\mathrm{tl})}$. 
For efficient acceleration each trailing microbunch must be located at the point where the phase of the saturated wake is nearly $\pi(2 \kappa-1)(\kappa=1,2,3, \ldots)$. If for simplicity sake it is assumed that the phase of the saturated wake is zero, then it is required that $J_{1}\left(p_{s_{0}}\right)>0$. In addition, for the wake of the trailing bunch it is assumed that $J_{c}\left(\chi_{s_{0}}\right)>0$. Thus, efficient acceleration can be obtained when the spatial spread of each trailing microbunch, $\Delta z^{(\mathrm{tl})}$, is assumed to be shorter than the resonance wavelength $\left[\omega_{0} \Delta z^{(\mathrm{tl})} /(\beta c) \ll 1\right]$ and the location of the first microbunch satisfies $\omega_{0} z_{1}^{(\mathrm{tl})} /(\beta c)=\pi(2 \kappa-1)$.

In this configuration, the efficiency of the AM-TBA for trailing bunch traverses distance $L$ longer than the saturation length, $L_{\text {sat }}$, is defined by

$$
\eta \equiv \frac{W_{\mathrm{b}} L}{W_{\mathrm{em}}\left(T=T_{\mathrm{sat}}\right)\left(L+L_{\mathrm{sat}}\right)} \simeq \frac{W_{\mathrm{b}}}{\frac{1}{2} \hbar \omega_{0} \pi R^{2} n_{0}},
$$

where $W_{\mathrm{b}}$ is the total energy exchange (per unit length) for the accelerating trailing bunch, $W_{\mathrm{em}}\left(T=T_{\text {sat }}\right)$ is the stored electromagnetic energy after the complete depletion of the PID and in absence of the trailing bunch. Thus, to calculate the efficiency, we need to calculate the total energy exchange.

The total energy exchange, $W_{\mathrm{b}}$,

$$
\begin{aligned}
W_{\mathrm{b}} & =W_{\mathrm{b}}^{(\mathrm{sat})}+W_{\mathrm{b}}^{(\mathrm{ll})} \\
& =\int_{0}^{2 \pi} d \phi \int_{0}^{R} r d r \int_{-\infty}^{\infty} d T J_{z}\left(E_{\mathrm{sat}}+E_{z}^{(\mathrm{tl})}\right)
\end{aligned}
$$

is the sum of the energy exchange due to the saturated field $\left(W_{\mathrm{b}}^{(\mathrm{sat})}\right)$ and due to the accelerating trailing bunch $\left(W_{\mathrm{b}}^{(\mathrm{tl})}\right)$. For $W_{\mathrm{b}}>0$ energy is transferred from the electromagnetic fields as a kinetic energy to the accelerating bunch and for $W_{\mathrm{b}}<0$ electromagnetic field is generated on the expense of the trailing bunch energy.

The energy exchange due to the saturated field for the accelerating trailing bunch is

$$
W_{\mathrm{b}}^{(\mathrm{sat})}=-e \sqrt{\frac{\hbar \omega_{0} n_{0}}{\epsilon_{0} \epsilon_{r}}} \frac{J_{c}\left(\chi_{s_{0}}\right)}{J_{1}\left(p_{s_{0}}\right)} \sum_{\nu=1}^{M^{(\mathrm{t})}} \sum_{m=1}^{N^{(\mathrm{t})}} \cos \left(\Psi_{m, \nu}\right) .
$$

In the limit of large number of experiments $\left(N^{(\exp )} \gg 1\right)$ and for uniform electron phase distribution we obtain with similar procedure as in Appendix A that

$$
W_{\mathrm{b}}^{(\mathrm{sat})}=e N^{(\mathrm{tl})} M^{(\mathrm{tl})} \sqrt{\frac{\hbar \omega_{0} n_{0}}{\epsilon_{0} \epsilon_{r}}} B_{s_{0}} \operatorname{sinc}\left(\alpha_{s_{0}}\right) \cos \left(\phi_{s_{0}}\right),
$$

where $\alpha_{s}=\omega_{s} \Delta z^{(\mathrm{tl})} /(2 \beta c)$ and $\delta_{s}=\omega_{s} \lambda_{0} /(2 \beta c)$. In addition, $B_{s} \equiv J_{c}\left(\chi_{s}\right) \operatorname{sinc}\left(\delta_{s} M^{(\mathrm{tl})}\right) /\left[\operatorname{sinc}\left(\delta_{s}\right) J_{1}\left(p_{s}\right)\right]$ and $\phi_{s_{0}}=\alpha_{s_{0}}\left(1+2 z_{1} / \Delta z^{(\mathrm{tl})}\right)+\pi+\delta_{s_{0}}\left(M^{(\mathrm{tl})}-1\right)$. Note that
$B_{s_{0}} \operatorname{sinc}\left(\alpha_{s_{0}}\right) \cos \left(\phi_{s_{0}}\right)>0$ under the previously mentioned assumptions of $J_{1}\left(p_{s_{0}}\right)>0, J_{c}\left(\chi_{s_{0}}\right)>0, \omega_{0} \Delta z^{(\mathrm{tl})} /(\beta c) \ll 1$, and $\omega_{0} z_{1}^{(\mathrm{tl})} /(\beta c)=\pi(2 \kappa-1)$. Hence, the energy exchange due to the saturated wake has a positive value. This means that it contributes to the acceleration of the trailing bunch.

The energy exchange due to the Cerenkov wake for the accelerating trailing bunch is

$$
\begin{aligned}
W_{\mathrm{b}}^{(\mathrm{tl})}= & -\frac{\left(e N^{(\mathrm{tl})} M^{(\mathrm{tl})}\right)^{2}}{\epsilon_{0} \epsilon_{r} \pi R^{2}} \frac{J_{c}^{2}\left(\chi_{s}\right)}{J_{1}^{2}\left(p_{s}\right)} \frac{1}{\left(M^{(\mathrm{tl})} N^{(\mathrm{tl})}\right)^{2}} \\
& \times \sum_{\nu, \nu^{\prime}=1}^{M^{(\mathrm{l})}} \sum_{m, m^{\prime}=1}^{N^{(\mathrm{tl})}} \cos \left[\frac{\omega_{s} \lambda_{0}}{2 \pi c}\left(\Psi_{m, \nu}-\Psi_{m^{\prime}, \nu^{\prime}}\right)\right] \\
& \times \Theta\left(\Psi_{m, \nu}-\Psi_{m^{\prime}, \nu^{\prime}}\right) .
\end{aligned}
$$

Thus, in the limit of large number of experiments $\left(N^{(\exp )} \gg 1\right)$ and for uniform electron phase distribution we obtain

$$
W_{\mathrm{b}}^{(\mathrm{tl})}=-\frac{\left(e N^{(\mathrm{tl})} M^{(\mathrm{tl})}\right)^{2}}{\epsilon_{0} \epsilon_{r} \pi R^{2}} \sum_{s=1}^{\infty} B_{s}^{2}\left[\frac{1+\operatorname{sinc}^{2}\left(\alpha_{s}\right)}{4}\right] .
$$

Thus, the energy exchange due to the Cerenkov wake of the trailing bunch has a negative value. This is an expected result since it contributes to the generation of electromagnetic field on the expense of the bunch kinetic energy. Therefore, the total energy exchange (per unit length) for the accelerating trailing bunch is

$$
W_{\mathrm{b}}=\sqrt{W_{\mathrm{b}, 1}} N^{(\mathrm{tl})}\left(1-N^{(\mathrm{tl})} f_{\mathrm{col}}\right),
$$

where

$$
W_{\mathrm{b}, 1} \equiv e M^{(\mathrm{tl})} \sqrt{\frac{\hbar \omega_{0} n_{0}}{\epsilon_{0} \epsilon_{\mathrm{r}}}} B_{s_{0}} \operatorname{sinc}\left(\alpha_{s_{0}}\right) \cos \left(\phi_{s_{0}}\right)
$$

is the energy transfer in case of trailing bunch with $M^{(\mathrm{tl})}$ microbunches so that each microbunch consists of a single electron and

$$
f_{\text {col }} \equiv \frac{e}{\sqrt{\epsilon_{0} \epsilon_{\mathrm{r}} \hbar \omega_{0} n_{0}} \pi R^{2}} \frac{M^{(\mathrm{ll})} \sum_{s=1}^{\infty} B_{s}^{2}}{B_{s_{0}} \operatorname{sinc}\left(\alpha_{s_{0}}\right) \cos \left(\phi_{s_{0}}\right)}
$$

is the collective-effect coefficient.

The total energy exchange given in Eq. (D11) has three important regimes. In the first regime, for a small number of electrons per microbunch $\left(N^{(\mathrm{tl})} \ll 1 / f_{\text {col }}\right)$ the total wakefield in the location of the bunch is nearly constant and equal to the saturated field. Since the number of electrons is small, the current is also small. Hence, the total energy exchange proportional to the product of the wakefield with the current will be small. In the second regime, for a large 
number of electrons in each microbunch $\left(N^{(\mathrm{tl})} \simeq 1 / f_{\text {col }}\right)$, however, the total wakefield will tend to zero and the current will be large; the overall result is small total energy exchange. Thus, between the two regimes there is an optimum number of electrons in each microbunch to obtain (the third regime) maximum energy exchange and efficiency. The maximum energy exchange for given number of microbunches is

$$
\begin{aligned}
W_{\mathrm{b}}^{(\max )} & =\frac{W_{\mathrm{b}, 1}}{4 f_{\mathrm{col}}} \\
& =\frac{1}{4} \hbar \omega_{0} n_{0} \pi R^{2} \frac{B_{s_{0}}^{2} \operatorname{sinc}^{2}\left(\alpha_{s_{0}}\right) \cos ^{2}\left(\phi_{s_{0}}\right)}{\sum_{s=1}^{\infty} B_{s}^{2}\left(\frac{1+\operatorname{sinc}^{2} \alpha_{s}}{4}\right)}
\end{aligned}
$$

and the optimum number of electrons in a microbunch is

$$
\begin{aligned}
N^{(\mathrm{tl}),(\mathrm{opt})}= & \frac{1}{2 f_{\mathrm{col}}}=\frac{\pi R^{2}}{2 e M^{(\mathrm{tl})}} \sqrt{\hbar \omega_{0} n_{0} \epsilon_{0} \epsilon_{r}} \\
& \times \frac{B_{s_{0}} \operatorname{sinc}\left(\alpha_{s_{0}}\right) \cos \left(\phi_{s_{0}}\right)}{\sum_{s=1}^{\infty} B_{s}^{2}\left(\frac{1+\operatorname{sinc}^{2} \alpha_{s}}{4}\right)} .
\end{aligned}
$$

The maximum efficiency which is found by substituting Eq. (D14) into Eq. (D5) is

$$
\eta^{(\max )}=\frac{B_{s_{0}}^{2} \operatorname{sinc}^{2}\left(\alpha_{s_{0}}\right) \cos ^{2}\left(\phi_{s_{0}}\right)}{\sum_{s=1}^{\infty} B_{s}^{2}\left(\frac{1+\operatorname{sinc}^{2} \alpha_{s}}{2}\right)} .
$$

Thus, maximum efficiency of $100 \%$ is obtained for $M^{(\mathrm{tl})} \gg$ 1 and a pointlike microbunch, $\omega_{0} \Delta z^{(\mathrm{tl})} /(\beta c) \rightarrow 0$. Since for large mode number $\left|\operatorname{sinc}\left(\delta_{s} M^{(\mathrm{tl})}\right) / \operatorname{sinc}\left(\delta_{s}\right)\right|=1$ at the high harmonics of $s_{0}$, the $100 \%$ efficiency is valid only for small resonance mode number where $\left|B_{s}\right|$ is maximal only at the resonance. In addition, the optimum $N^{(\mathrm{tl}),(\mathrm{opt})}$ is

$$
\begin{aligned}
N^{(\mathrm{tl}),(\mathrm{opt})}\left(M^{(\mathrm{ll})} \gg 1\right) \simeq & \frac{\pi R^{2}}{e M^{(\mathrm{tl})}} \frac{J_{1}\left(p_{s_{0}}\right)}{J_{c}\left(\chi_{s_{0}}\right)} \\
& \times \sqrt{\hbar \omega_{0} n_{0} \epsilon_{0} \epsilon_{r}} .
\end{aligned}
$$

\section{APPENDIX E: EMITTANCE GROWTH FROM COULOMB SCATTERING}

The emittance growth due to Coulomb scattering is [19]

$$
\frac{d \varepsilon}{d z}=\frac{1}{2} \frac{\gamma}{k_{\beta}} \frac{d}{d z}\left\langle\theta^{2}\right\rangle
$$

where $\langle\theta\rangle$ is the mean-square scattering angle for single collision projected in one plane and $k_{\beta}$ is the betatron wave number. The betatron wave number is $k_{\beta}=\frac{\omega_{\beta}}{\sqrt{\gamma} c}$, where the squared betatron frequency is $\omega_{\beta}^{2}=-\left.\frac{1}{m} \frac{\partial F}{\partial r}\right|_{r=0}$. Hence, $k_{\beta}^{2}=\left.\frac{1}{m_{e} c^{2} \gamma} \frac{\partial F_{r}}{\partial r}\right|_{r=0}$, where $F_{r}=-e\left(E_{r}-c \mu_{0} H_{\phi}\right)$ is the radial force. In the blowout regime of plasma-based accelerators, an ion channel is formed which is used for electron beam focusing. In plasma wakefield accelerator (PWFA) the betaron wave number is $k_{\beta}=\frac{k_{p}}{\sqrt{2 \gamma}}$ [20], where $k_{p}=\sqrt{n_{p} e^{2} /\left(m_{e} \epsilon_{0} c^{2}\right)}$ is the plasma wave number and $n_{p}$ is the plasma density. The growth of the scattering angle from electron ion channel collision is [20]

$$
\frac{d\left\langle\theta^{2}\right\rangle_{\mathrm{PWFA}}}{d z}=\frac{k_{p}^{2} r_{e}}{\gamma^{2}} S,
$$

where $r_{e}$ is the classical electron radius and

$$
S=Q\left[\ln \left(\frac{R_{b}}{R_{a}}\right)+\frac{1.78 Z(Z+1)}{Q^{2}} \ln \left(\frac{287}{\sqrt{Z}}\right)\right]
$$

is a parameter that depends on the ion channel geometry, $Z$ is the atomic number, $Q=n_{p} / n, n$ is the density of a neutral vapor, $R_{b}$ is the blow out radius, and $R_{a}$ is the atomic radius. After substituting the betatron wave number and growth of the scattering angle [Eq. (E2)] into Eq. (E1), one obtains that the growth of emittance from Coulomb scattering in PWFA reads

$$
\frac{d \varepsilon_{\mathrm{PWFA}}}{d z}=\frac{k_{p}}{\sqrt{2 \gamma}} r_{e} S .
$$

It is more practical to calculate the growth of the emittance as a result of beam acceleration, namely growth in $\gamma$. Hence, $\frac{d \varepsilon}{d \gamma}=\frac{k_{p} r_{e} S}{\dot{\gamma} \sqrt{2 \gamma}}$, where $\dot{\gamma}=d \gamma / d z$. In PWFA, $d \gamma / d z=k_{p}$ hence the growth of the emittance is

$$
\Delta \varepsilon_{\mathrm{PWFA}}=\sqrt{2} r_{e} S\left(\sqrt{\gamma_{f}}-\sqrt{\gamma_{i}}\right) .
$$

In our structure, the radial force at saturation is $F_{r}=e \frac{\left(1-\beta \varepsilon_{r}\right) \omega}{\beta k_{s} c} E_{\mathrm{sat}} J_{1}\left(k_{s} r\right) \sin (\omega T)$, where the longitudinal wakefield is assumed to be $E_{z}=E_{\text {sat }} J_{0}\left(k_{s} r\right) \cos (\omega T)$. Hence, similarly as in a plasma based accelerator, the generated wakefield can be used for both accelerating and focusing the beam. Near the axis the radial force $F_{r}(r \approx 0)=-e E_{\text {sat }} \frac{\omega}{2 c} \sqrt{\bar{\varepsilon}} r \sin (\omega T)$ where the resonance condition was used. In addition, the growth of the scattering angle from electron vapor collisions is [28]

$$
\frac{d\left\langle\theta^{2}\right\rangle_{\text {vapor }}}{d z}=\frac{\rho}{2 \gamma^{2} X_{0}}\left(\frac{14 \mathrm{MeV}}{m_{e} c^{2}}\right)^{2},
$$

where $X_{0}$ is the radiation length measured in $\mathrm{g} / \mathrm{cm}^{2}$ and $\rho$ is the weight density of the gas mixture measured in $\mathrm{g} / \mathrm{cm}^{3}$ which can estimated from the ideal gas law. Hence, the emittance growth due to Coulomb scattering in AM-TBA is

$$
\begin{aligned}
\frac{d \varepsilon_{\mathrm{AM}-\mathrm{TBA}}}{d z}= & \frac{\sqrt{m_{e} c^{2} / e}}{2 \sqrt{2}}\left(\frac{14 \mathrm{MeV}}{m_{e} c^{2}}\right)^{2} \frac{\rho}{X_{0}} \\
& \times \frac{1}{\sqrt{\gamma\left|E_{\mathrm{sat}}\right|(\omega / c) \sqrt{\bar{\varepsilon}}}}
\end{aligned}
$$


where the value of the wake at saturation is given in Eq. (12). Similarly as in the PWFA the growth of the emittance from acceleration is

$$
\begin{aligned}
\Delta \varepsilon_{\mathrm{AM}-\mathrm{TBA}}= & \frac{1}{2}\left(\frac{14 \mathrm{MeV}}{m_{e} c^{2}}\right)^{2} \frac{\rho}{X_{0}}\left(\frac{m_{e} c^{2} / e}{\left|E_{\mathrm{sat}}\right|}\right)^{1.5} \frac{1}{\sqrt{(\omega / c) \sqrt{\bar{\varepsilon}}}} \\
& \times\left(\sqrt{\gamma_{f}}-\sqrt{\gamma_{i}}\right),
\end{aligned}
$$

where in AM-TBA $\frac{d \gamma}{d z}=\frac{\left|E_{\text {sat }}\right|}{m_{e} c^{2} / e}$.

[1] T. P. Wangler, RF Linear Accelerators, 2nd ed. (WileyVCH, New York, 2008).

[2] R. B. Neal, The Stanford Two-Mile Accelerator (Benjamin, New York, 1968).

[3] T. Behnke, J. E. Brau, B. Foster, J. Fuster, M. Harrison, J. M. Paterson, M. Peskin, M. Stanitzki, N. Walker, and H. Yamamoto, arXiv:1306.6327.

[4] P. Sprangle and B. Hafizi, Phys. Plasmas 21, 055402 (2014).

[5] E. Esarey, C. B. Schroeder, and W. P. Leemans, Rev. Mod. Phys. 81, 1229 (2009).

[6] S. M. Hooker, Nat. Photonics 7, 775 (2013).

[7] E. A. Peralta, K. Soong, R. J. England, E. R. Colby, Z. Wu, B. Montazeri, C. McGuinness, J. McNeur, K. J. Leedle, D. Walz, E. B. Sozer, B. Cowan, B. Schwartz, G. Travish, and R. L. Byer, Nature (London) 503, 91 (2013).

[8] I. Blumenfeld, C. E. Clayton, F.-J. Decker, M. J. Hogan, C. Huang, R. Ischebeck, R. Iverson, C. Joshi, T. Katsouleas, N. Kirby, W. Lu, K. A. Marsh, W. B. Mori, P. Muggli, E. Oz, R. H. Siemann, D. Walz, and M. Zhou, Nature (London) 445, 741 (2007).

[9] L. Schachter, Phys. Rev. Lett. 83, 92 (1999).

[10] L. Schächter, Phys. Lett. A 277, 65 (2000).
[11] S. Banna, V. Berezovsky, and L. Schächter, Phys. Rev. Lett. 97, 134801 (2006).

[12] M. Voin and L. Schächter, Phys. Rev. Lett. 112, 054801 (2014).

[13] Z. Toroker, M. Voin, and L. Schächter, High Power Laser Sci. Eng. 2, e29 (2014).

[14] H. Wiedemann, Particle Accelerator Physics, 3rd ed. (Springer, New York, 2007), p. 559.

[15] M. Voin, W. D. Kimura, and L. Schächter, Nucl. Instrum. Methods Phys. Res., Sect. A 740, 117 (2014).

[16] P. Michel, C. B. Schroeder, B. A. Shadwick, E. Esarey, and W. P. Leemans, Phys. Rev. E 74, 026501 (2006).

[17] T. Mehrling, J. Grebenyuk, F. S. Tsung, K. Floettmann, and J. Osterhoff, Phys. Rev. ST Accel. Beams 15, 111303 (2012).

[18] I. Dornmair, K. Floettmann, and A. R. Maier, Phys. Rev. ST Accel. Beams 18, 041302 (2015).

[19] B. W. Montague and W. Schnell, AIP Conf. Proc. 130, 146 (1985).

[20] N. Kirby, M. Berry, I. Blumenfeld, M. Hogan, R. Ischebeck, and R. Siemann, in Proceedings of the 22nd Particle Accelerator Conference, PAC-2007, Albuquerque, NM (IEEE, New York, 2007), p. 3097.

[21] I. Y. Dodin and N. J. Fisch, Phys. Plasmas 15, 103105 (2008).

[22] D. Haberberger, S. Tochitsky, and C. Joshi, Opt. Express 18, 17865 (2010).

[23] M. N. Polyanskiy, I. V. Pogorelsky, and V. Yakimenko, Opt. Express 19, 7717 (2011).

[24] K. A. Olive et al., Chin. Phys. C 38, 090001 (2014).

[25] M. Voin and L. Schächter, Phys. Rev. Lett. 112, 054801 (2014).

[26] M. Voin and L. Schächter, Phys. Rev. ST Accel. Beams 18, 071302 (2015).

[27] V. M. Malkin, G. Shvets, and N. J. Fisch, Phys. Rev. Lett. 82, 4448 (1999).

[28] G. R. Lynch and O. I. Dahl, Nucl. Instrum. Methods Phys. Res., Sec B 58, 6 (1991). 